\title{
SOMMES DE CESÀRO ET MULTIPLICATEURS DES DÉVELOPPEMENTS EN HARMONIQUES SPHÉRIQUES
}

\author{
PAR
}

\author{
ALINE BONAMI ET JEAN-LOUIS CLERC
}

\begin{abstract}
Nous établissons une inégalité entre les sommes de Cesàro et la fonction maximale associées à une fonction définie sur la sphère, et nous en déduisons divers résultats de convergence en norme $L^{p}$, convergence presque partout, localisation des développements en harmoniques sphériques, ainsi qu'un théorème de multiplicateurs qui généralise le théorème classique de Marcinkiewicz sur les séries trigonométriques. La même étude est faite pour les développements suivant les polynômes ultrasphériques. Nous montrons de plus que les sommes partielles du développement en harmoniques sphériques d'une fonction de $L^{p}\left(\Sigma_{n}\right)$, $p \neq 2$, ne convergent pas forcément en norme.
\end{abstract}

Le théorème classique des multiplicateurs de Marcinkiewicz s'énonce (voir [16]):

Théorème (A). Soit $\left(\mu_{k}\right)_{k \geq 0}$ une suite de nombres complexes, telle que $\left(\mathrm{A}_{0}\right)\left|\mu_{k}\right| \leq A$

$\left(A_{1}\right) \sup _{j} \Sigma_{2 j}^{2^{j+1}}\left|\mu_{k+1}-\mu_{k}\right| \leq A$.

Alors $\left\|\Sigma_{0}^{+\infty} \mu_{k}\left(a_{k} \cos k \theta+b_{k} \sin k \theta\right)\right\|_{p} \leq A_{p}\left\|\Sigma_{0}^{+\infty}\left(a_{k} \cos k \theta+b_{k} \sin k \theta\right)\right\|_{p}$, $1<p<\infty$.

Le but de ce travail est de généraliser ce résultat aux développements en harmoniques sphériques. Plus précisément, si $f=\Sigma_{0}^{+\infty} H_{k} f$ est la décomposition en harmoniques sphériques d'une fonction $f$ sur la sphère $\Sigma_{n}$, on cherche des conditions suffisantes (de type analogue à celles du Théorème $(A)\left({ }^{1}\right)$ ) sur la suite $\left(\mu_{k}\right)_{k \geq 0}$ pour que l'application linéaire $M$ définie par $M f=\Sigma_{0}^{+\infty} \mu_{k} H_{k} f$ soit continue de $L^{p}$ dans $L^{p}(1<p<+\infty)$.

La démonstration de (A) utilise un certain nombre d'outils, et principalement les deux théorèmes suivants $\left(S_{N} f\right.$ désignant la Nième somme partielle, et $P_{r}(0 \leq r<1)$ le noyau de Poisson):

Received by the editors July 19, 1972.

AMS (MOS) subject classifications (1970). Primary 43A85, 43A55, 43A22; Secondary 40G05, 42A56.

Key words and phrases. Spherical harmonics, Cesàro means, summability, localization, $L^{p}$ multipliers, ultraspherical polynomials。

(1) R. Coifman et G. Weiss ont obtenu dans [5] des conditions suffisantes "de type Hörmander". Nos résultats sont indépendants des leurs et strictement plus forts dans ce cas. 
Théorème (B) (fonction $g$ de Littelwood-Paley). Il existe, pour $1<p<+\infty$, une constante $A_{p}$, telle que

(i) $\|g(f)\|_{p}=\left\|\left(\int_{0}^{1}(1-r)\left|\partial P_{r} / \partial r * f\right|^{2} d r\right)^{1 / 2}\right\|_{p} \leq A_{p}\|f\|_{p}$;

(ii) $\|f\|_{p} \leq A_{p}\|g(f)\|_{p}$ si $\int_{T} f(x) d x=0$.

Théorème (C) (inégalité de Zygmund). Il existe, pour $1<p<+\infty$, une cons tante $A_{p}$, telle que, pour toute suite d'entiers, $n_{1}, n_{2}, \cdots, n_{k}, \cdots$ et toute suite de fonctions $f_{1}, f_{2}, \cdots, f_{k}, \cdots$

$$
\left\|\left(\sum_{0}^{+\infty}\left|S_{n_{k}} f_{k}(x)\right|^{2}\right)^{1 / 2}\right\|_{p} \leq A_{p}\left\|\left(\sum_{0}^{+\infty}\left|f_{k}(x)\right|^{2}\right)^{1 / 2}\right\|_{p} .
$$

Une généralisation très complète de (B) a été obtenue par Stein [19]; il montre que si $\left(T^{t}\right)_{t \geq 0}$ est un semi-groupe de diffusion symétrique sur un espace de mesure $M$, id est

(i) $T^{t+s}=T^{t} \circ T^{s}, T^{0}=\mathrm{Id}$,

(ii) $\left\|T^{t} f\right\|_{p} \leq\|f\|_{p}$ pour $1 \leq p \leq+\infty$, et $T^{t} f \rightarrow f$ dans $L^{2}(\mathbb{N})$ quand $t \rightarrow 0$,

(iii) $T^{t}$ est un opérateur auto-adjoint sur $L^{2}(\mathbb{N})$,

(iv) $f \geq 0 \Rightarrow T^{t} f \geq 0$,

(v) $T^{t} 1=1$,

alors la fonction $g$ définie par $g(f)(x)=\left(\int_{0}^{+\infty} t\left|(\partial / \partial t) T^{t} f(x)\right|^{2} d t\right)^{1 / 2}$ satisfait l'analogue du Théorème (B). Stein en déduit d ailleurs un théorème de multiplicateurs, qui, dans un cadre aussi général, est le meilleur auquel on puisse s'attendre.

Dans le cas des variétés riemanniennes compactes, le semi-groupe de la chaleur, dont le générateur infinitésimal est l'opérateur de Laplace-Beltrami satisfait les conditions (i) à (v). On obtient donc un théorème de multiplicateurs, mais il réclame des conditions sur une infinité de différences. Stein pose le problème d'une généralisation plus satisfaisante de (A) dans ce cas.

Un substitut de l'inégalité (C) est fourni par le résultat suivant, cas particulier d'un résultat de Fefferman et Stein(2).

Théorème (D). Soit $(\mathbb{M}, d)$ un espace métrique, muni d'une mesure $\mu$ satisfaisant $\mu(B(x, r)) \leq C \cdot \mu(B(x, r / 2))$. On note $M f$ la fonction maximale de Hardy. Littlewood

$$
M f(x)=\sup _{r>0}(1 / \mu(B(x, r))) \int_{B(x, r)}|f(y)| d \mu(y) .
$$

Il existe alors, pour $1<p<\infty$, une constante $A_{p}$, telle que pour toute suite $f_{1}, f_{2}, \cdots, f_{k}, \cdots$

(2) Le résultat est démontré pour $\mathbf{R}^{n}$ dans [8]; il se généralise facilement aux "espaces de nature homogène" (pour cette notion voir [5, Chapitre III]). 


$$
\left\|\left(\sum_{0}^{+\infty}\left|M f_{k}(x)\right|^{2}\right)^{1 / 2}\right\|_{p} \leq A_{p}\left\|\left(\sum_{0}^{+\infty}\left|f_{k}(x)\right|^{2}\right)^{1 / 2}\right\|_{p}
$$

Pour obtenir la généralisation de (C) dans le cas des sphères, on doit d'abord recourir à un procédé de sommation plus efficace: nous montrons en effet que les sommes partielles des développements en harmoniques sphériques ne sont pas uniformément bornées dans $L^{p}\left(\Sigma_{n}\right)$, sauf si $p=2$ (voir $\$ 5$ ). On utilise les sommes de Cesàro, dont on rappelle qu'elles sont défines par

$$
S_{L}^{\delta} f=\frac{1}{A_{L}^{\delta}} \sum_{0}^{L} A_{L-l}^{\delta} H_{l} f, \quad \text { où } A_{L}^{\delta}=\frac{(l+\delta) \cdots(\delta+1)}{l(l-1) \cdots 1} .
$$

Si $\delta>(n-1) / 2$, il était connu que les sommes de Cesàro sont uniformément bornées dans $L^{p}(1 \leq p \leq+\infty)$. Nous donnons ici une estimation ponctuelle de $\sup _{L}\left|S_{L}^{\delta} f\right|$ à l'aide précisément de la fonction maximale de Hardy-Littlewood, ce qui permet d'obtenir le résultat (C) moyennant (D).

Une fois obtenus les résultats $(B)$ et $(C)$, nous en déduisons un théorème de multiplicateurs, en généralisant convenablement la méthode utilisée par Muckenhoupt et Stein (voir [17]), dans leur étude des développements suivant les polynômes ultrasphériques.

Notre théorème principal s'énonce alors:

Théorème (0.1). Soit $\left(\mu_{k}\right)_{k \geq 0}$ une suite de nombres complexes satisfaisant aux conditions

$$
\begin{aligned}
& \left(A_{0}\right)\left|\mu_{k}\right| \leq C, \\
& \left(A_{N}\right) \sup _{j} 2^{j(N-1)} \Sigma_{2^{j}}^{2^{j+1}}\left|\Delta^{N} \mu_{k}\right| \leq C,
\end{aligned}
$$

où $N$ est le premier entier strictement plus grand que $n / 2$, alors

$$
\left\|\sum \mu_{k} H_{k} f\right\|_{L^{p}\left(\Sigma_{n}\right)} \leq A_{p}\left\|\sum H_{k} f\right\|_{L^{p}\left(\Sigma_{n}\right)} \quad(1<p<+\infty) .
$$

En résumé, on peut donner la marche à suivre pour la démonstration d'un théorème de multiplicateurs (type Marcinkiewicz). On étudie la convergence en moyenne dans $L^{p}(1 \leq p<+\infty)$ des sommes de Cesàro (ou des sommes de Riesz), pour laquelle apparait un indice critique $\delta_{0}$, qui détermine en quelque sorte la "dimension harmonique" du système orthogonal en cause. On cherche alors, par des estimations plus fines, à démontrer une "inégalité maximale", d'où découle (C). Notre conjecture est que l'indice critique pour le Théorème (C) est encore $\delta_{0}$. On en déduit alors un théorème de multiplicateurs dans $L^{p}(1<p<+\infty)$, qui fait intervenir $N$ différences (ou dérivées), où $N$ est le premier entier supérieur ou égal à $\delta_{0}+1$. Nous indiquons d'ailleurs au $\$ 7$ des généralisations de nos résultats au cas des espaces symétriques compacts de rang 1 , des variétés riemanniennes compactes, et au $\$ 6$, au cas des polynômes ultrasphériques. 
La conjecture que le nombre de différences qui intervient est lié à l'indice critique des sommes de Cesàro se voit confirmée dans un autre résultat que nous obtenons, et qui établit un lien entre les multiplicateurs de $\Sigma_{n}$, et les multiplicateurs radiaux de $\mathbf{R}^{n}$. Si ces liens sont sans doute moins complets que ceux existant entre les multiplicateurs de $T^{n}$ et ceux de $\mathbf{R}^{n}$, ils permettent de trouver des résultats négatifs sur $\Sigma_{n}$, tels que la non convergence des sommes partielles, ou des résultats positifs sur $\mathbf{R}^{n}$ : c'est le cas du théorème de multiplicateurs suivant (qu'on pourrait également obtenir directement), qui n'est conséquence ni du théorème d'Hörmander, ni du théorème de Marcinkiewicz.

Théorème (0.2). Soit $m$ une fonction à valeurs complexes, de classe $\mathcal{C}^{N}$ sur ]0, $+\infty)$, et satisfaisant aux conditions:

$$
\begin{aligned}
& \left(\mathrm{A}_{0}\right)|m(\rho)| \leq C, \\
& \left(\mathrm{~A}_{N}\right) 2^{j(N-1)} \int_{2^{j}}^{2^{j+1}}\left|d^{N} m(\rho) / d \rho^{N}\right| d \rho \leq C,
\end{aligned}
$$
où $N$ est le premier entier strictement plus grand que $n / 2$. Alors $m$ définit un multiplicateur radial de $L^{p}\left(\mathbf{R}^{n}\right)(1<p<+\infty)$.

En outre, nous donnons une étude systématique des sommes de Cesàro: résultats de convergence presque partout et localisation (on comparera utilement avec les résultats de Stein [18] sur $\mathrm{T}^{n}$ ), étude en-dessous de l'indice critique (cf. les résultats de Fefferman [6]). Nous donnons aussi les théorèmes semblables pour les développements suivant les polynômes ultrasphériques, étendant ainsi les résultats de Muckenhoupt et Stein [17], Askey-Hirschman [1] et Gilbert [10].

Certains des résultats qui figurent ici ont fait l'objet de notes aux C. R. Acad. Sci. ([2], [3], [4]).

Cette introduction serait incomplète, si nous ne remerciions E. M. Stein, qui, de séjour à Orsay nous a accueilli avec bienveillance, nous a éclairé de ses intuitions sur ces problèmes, et nous a communiqué oralement une démonstration simplifiée des résultats de Fefferman [6]. Nous avons également bénéficié de l'enseignement dispensé au cours de l'année 1970-1971 par R. Coifman et $\mathrm{G}$. Weiss, qui nous ont en particulier initiés à la théorie des intégrales singulières sur les espaces de nature homogène. N. Lohoué nous a toujours amicalement encouragés et nous a en particulier suggéré l'énoncé du théorème (1.1). Enfin Madame Dumas, avec sa compétence habituelle, a fait de notre manuscrit quelque peu indigeste un article lisible-du moins nous l'espérons.

1. Lien entre les multiplicateurs zonaux de $\Sigma_{n}$ et les multiplicateurs radiaux de $\mathrm{R}^{n}$. On note $\Sigma_{n}$ la sphère de rayon 1 dans $\mathrm{R}^{n+1}$, id est $\Sigma_{n}=$ $\left\{\xi \in \mathbf{R}^{n+1},\|\xi\|^{2}=1\right\} ; 1$ désigne le pôle nord de coordonnées $(0,0, \cdots, 0,1)$. 
La sphère est munie de la métrique riemannienne induite par celle de $R^{n+1}$. On note exp l'application exponentielle au pôle nord, $d$ la distance géodésique sur $\Sigma_{n}$, et $d \sigma$ la mesure riemannienne, normalisée de sorte que $\int_{\Sigma_{n}} d \sigma=1$. Son expression en coordonnées exponentielles est $A_{n}((\sin \|x\|) /\|x\|)^{n-1} d x$ 。

$\Sigma_{n}$ peut aussi être considérée comme l'espace homogène $S O(n+1) / S O(n)$, où $S O(n)$ est le sous-groupe de $S O(n+1)$ qui laisse le pôle nord invariant. Une fonction à valeurs complexes $f$ sur $\Sigma_{n}$ s'identifie à une fonction ${ }^{\sharp} f$ sur $S O(n+1)$, constante sur les classes d'équivalence, au moyen de ${ }^{\sharp} f(g)=f(g(1))$. Cela permet de définir une structure de convolution sur $\Sigma_{n}$. Une classe particulière de fonctions, dites zonales, est constituée des fonctions $f$ sur $\Sigma_{n}$, invariantes par l'action de $S O(n)$. Une telle fonction ne dépend alors que de la distance géodésique au pôle nord (3), ou encore du produit scalaire (dans $\mathbf{R}^{n+1}$ ), soit $f(\xi)=$ ${ }^{b} f(\xi \cdot 1)$, où bf est défini sur le segment $[-1,+1]$. La convolution d'une fonction zonale $f$ avec une fonction quelconque $g$ s'écrit alors simplement:

$$
(f * g)(\xi)=\int_{\Sigma_{n}} b_{f}(\xi \cdot \eta) g(\eta) d \sigma(\eta) .
$$

Les sous-espaces de $L^{2}\left(\Sigma_{n}\right)$ invariants minimaux sous l'action de $S O(n+1)$ sont les sous-espaces $\mathcal{H}_{k}(k \in \mathbf{N})$, formés des harmoniques sphériques homogènes de degré $k_{\text {。 }}$ On note $H_{k}$ le projecteur orthogonal sur $\mathcal{H}_{k^{\prime}}$ et $\mathrm{Z}_{k}$ l'unique fonction zonale de $\mathcal{H}_{k}$, telle que

$$
\forall \phi \in \mathcal{H}_{k}, \quad\left\langle\phi, \mathrm{Z}_{k}\right\rangle=\phi(1) .
$$

Il est classique (voir [5], [23]) que $f=\Sigma_{0}^{+\infty} H_{k} f\left(f \in L^{2}\left(\Sigma_{n}\right)\right.$ ), que $H_{k} f=\mathbf{Z}_{k} * f$, et que ${ }^{b} \mathrm{Z}_{k}=(n-1+2 k) /(n-1) P_{k}^{\lambda}(\lambda=(n-1) / 2)$, où $P_{k}^{\lambda}$ est le polynôme de de Gegenbauer d'indice $\lambda$ et de degré $k$, défini par $\left(1-2 x w+w^{2}\right)^{-\lambda}=\Sigma_{0}^{+\infty} P_{k}^{\lambda}(x) w^{k}$. On en déduit aisément le noyau de Poisson de la sphère $\Sigma_{n}$ :

$$
P_{w}(\xi)=\sum_{0}^{+\infty} w^{k} \mathbf{Z}_{k}(\xi)=\frac{1-w^{2}}{\left(1-2 w\langle\xi \cdot 1\rangle+w^{2}\right)^{(n+1) / 2}}
$$

On appelle multiplicateur zonal de $L^{2}\left(\Sigma_{n}\right)$ tout opérateur de $L^{2}\left(\Sigma_{n}\right)$ qui commute à l'action de $S O(n+1)$. Un tel opérateur $M$ s'écrit sous la forme $M f=$ $\Sigma_{0}^{+\infty} m_{k} H_{k} f$, où $\left(m_{k}\right)_{k \geq 0}$ est une suite bornée de nombres complexes. Si $M$ s'étend en un opérateur continu de $L^{p}$ dans $L^{p}$, on dit que $M$ est un multiplicateur de $L^{p}\left(\Sigma_{n}\right)$. Cet opérateur peut aussi s'interpréter comme l'opérateur de convolution avec la distribution $\Sigma_{0}^{+\infty} m_{k} \mathrm{Z}_{k}$.

On sera amené à envisager dans la suite la sphère de rayon $R$ dans $R^{n+1}$; les notations $\Sigma_{R}$, $\exp _{R}, d \sigma_{R}$ se laissent comprendre par analogie avec la sphère-unité.

(3) Ceci est faux pour $n=1$, il convient alors de se limiter aux fonctions paires sur le tore $S^{1}$. 
On se propose maintenant de rattacher les multiplicateurs zonaux de la sphère aux multiplicateurs radiaux de $R^{n}$. Dans le cas du tore $T^{n}$, l'isomorphisme local entre $T^{n}$ et $\mathrm{R}^{n}$ permet d'établir de nombreux résultats; la démonstration de l'un de ces résultats utilise une méthode de passage à la limite, que nous avons pu adapter aux cas des sphères. Une formule asymptotique pour la fonction zonale fournit alors le résultat désiré.

Soit donc $m$ une fonction mesurable bornée de $[0,+\infty)$ dans C. Une telle fonction permet de définir un multiplicateur radial $M$ dans $L^{2}\left(\mathbf{R}^{n}\right)$ par

$$
\widehat{M f}(x)=m(\|x\|) \hat{f}(x) .
$$

Par ailleurs, à chaque $\epsilon>0$, on associe la suite $m_{\epsilon}$ définie par $m_{\epsilon}(k)=m(\epsilon k)$, et on définit un multiplicateur $M_{\epsilon}$ dans $L^{2}\left(\Sigma_{n}\right)$ par $M_{\epsilon} f=\Sigma_{0}^{+\infty} m_{\epsilon}(k) H_{k} f$.

Théorème (1.1). On suppose que $m$ est une fonction bornée et continue à gauche en tout point de ]0, $+\infty\left[\right.$, que $M_{\epsilon}$ est pour chaque $\epsilon>0$ un multiplicateur de $L^{p}\left(\Sigma_{n}\right)$, et que $\sup _{\epsilon>0}\left\|M_{\epsilon}\right\|_{\mathfrak{L}\left(L^{p}\left(\Sigma_{n}\right)\right)} \leq A<+\infty$. Alors $M$ est un multiplicateur de $L^{p}\left(\mathbf{R}^{n}\right)$ et $\|M\|_{\mathcal{L}\left(L^{p}\left(\mathbf{R}^{n}\right)\right)} \leq C_{n} \cdot A$, òu $C_{n}$ ne dépend que de la dimension $n$.

Un raisonnement habituel montre qu'il suffit de démontrer le théorème pour des fonctions $m$ convenablement décroissantes à l'infini. En effet, posons $m^{t}(\rho)=m(\rho) e^{-t \rho}(t>0)$. Alors $m_{\epsilon}^{t}(k)=m_{\epsilon}(k) \cdot\left(e^{-\epsilon t}\right)^{k}=m_{\epsilon}(k) w^{k} ;$ or $\Sigma_{0}^{+\infty} w^{k} \mathbf{Z}_{k}$ est le noyau de Poisson $P_{w}$ de $\Sigma_{n}$, dont on sait qu'il est de norme 1 dans $L^{1}\left(\Sigma_{n}\right)$. Par suite $\left\|M_{\epsilon}^{i}\right\|_{\mathcal{L}\left(L^{p}\left(\Sigma_{n}\right)\right)} \leq\left\|M_{\epsilon}\right\|_{\mathcal{L}\left(L^{p}\left(\Sigma_{n}\right)\right)} \leq A$. Si donc le théorème est démontré pour $m^{t}$, on en déduit $\left\|M^{t}\right\|_{\mathcal{L}\left(L^{p}\left(\mathrm{R}^{n}\right)\right)} \leq C_{n} \cdot A$, d'où le résultat en notant que $m^{t} \rightarrow m$ en chaque point quand $t$ tend vers 0 . On peut donc supposer que $|m(\rho)| \leq C_{1} e^{-C_{2} \rho}, C_{1}, C_{2}>0$. Pour démontrer que $M$ est un opérateur de $L^{p}\left(\mathbf{R}^{n}\right)$ de norme inférieure à $C_{n} \cdot A$, il suffit de montrer que pour toutes fonctions $f$ et $g$ nulles hors d'un compact de $\mathbf{R}^{n}$, et telles que $\|f\|_{p} \leq 1,\|g\|_{p^{\prime}} \leq 1\left(1 / p+1 / p^{\prime}=1\right)$, on a l'inégalité $|I|=|\langle M f, g\rangle| \leq C_{n} A$. Mais $M$ n'est autre que la convolution avec la fonction radiale dont le profil est la transformée de Fourier-Bessel de $m$; le premier me mbre de l'inégalité s'écrit donc

$$
I=C_{n} \int_{\mathbf{R}^{n}} \int_{\mathbf{R}^{n}} \int_{0}^{+\infty}(\|x-y\| \rho)^{-\lambda+1 / 2} J_{\lambda-1 / 2}(\rho\|x-y\|) m(\rho) f(x) g(y) \rho^{n-1} d \rho d x d y,
$$

et l'intégrale est absolument convergente grâce au contrôle à l'infini de $m$.

Si $R$ est choisi suffisamment grand, $\exp _{R}: \mathrm{R}^{n} \rightarrow \Sigma_{R}$ est un difféomorphisme d'un voisinage ouvert $\Omega$ des supports de $f$ et $g$ sur un ouvert de $\Sigma_{R}$. Par suite, on peut définir sans ambiguité des fonctions $f_{R}$ et $g_{R}$ sur $\Sigma_{R}$, telles que 
$f_{R}\left(\exp _{R} x\right)=f(x), g_{R}\left(\exp _{R} x\right)=g(x)$, pour $x \in \Omega$. A l'aide de l'homothétie de rapport $1 / R$, et en utilisant l'hypothèse du théorème avec $\epsilon=1 / R$, on obtient l'inégalité suivante

$$
\left|I_{R}\right|=\left|\frac{1}{R^{n}} \int_{\Sigma_{R}} \int_{\Sigma_{R}} \sum_{0}^{+\infty} m\left(\frac{k}{R}\right)^{b} \mathbf{Z}_{k}\left(\frac{\xi^{\prime} \cdot \eta^{\prime}}{R^{2}}\right) f_{R}\left(\xi^{\prime}\right) g_{R}\left(\eta^{\prime}\right) d \sigma_{R}\left(\xi^{\prime}\right) d \sigma_{R}\left(\eta^{\prime}\right)\right| \leq A .
$$

On va montrer que $I_{R} \rightarrow C_{n} \cdot I$, quand $R$ tend vers $+\infty$. Par un changement de variables,

$$
\begin{aligned}
l_{R}=\frac{C^{n}}{R^{n}} \int_{\mathrm{R}^{n}} \int_{\mathrm{R}^{n}} & \sum_{0}^{+\infty} m\left(\frac{k}{R}\right){ }^{b} \mathrm{Z}_{k}\left(\frac{\exp _{R} x \cdot \exp _{R} y}{R^{2}}\right) \\
& \cdot f(x) g(y)\left(\frac{\sin \|x\| / R}{\|x\| / R}\right)^{n-1}\left(\frac{\sin \|y\| / R}{\|y\| / R}\right)^{n-1} d x d y .
\end{aligned}
$$

Posons pour un instant

$$
H_{R}(x, y)=\frac{1}{R^{n}} \sum_{0}^{+\infty} m\left(\frac{k}{R}\right)^{b} \mathrm{Z}_{k}\left(\frac{\exp _{R} x \cdot \exp _{R} y}{R^{2}}\right)=\int_{0}^{+\infty} b_{R}(\rho) \rho^{n-1} d \rho,
$$

où $b_{R}$ est la fonction sur $] 0,+\infty[$, constante par intervalles, définie comme suit: on appelle $k$ la partie entière de $R \rho(k \leq R \rho<k+1)$ et alors

$$
b_{R}(\rho)=\left(\int_{k / R}^{(k+1) / R} \rho^{n-1} d \rho\right)^{-1} \frac{1}{R^{n}} m\left(\frac{k}{R}\right)^{b} \mathrm{Z}_{k}\left(\frac{\exp _{R} x \cdot \exp _{R} y}{R^{2}}\right) .
$$

Utilisant la décroissance à l'infini de $m$, et le fait que ${ }^{b} \mathrm{Z}_{k}$ est $O\left(k^{n-1}\right)$, on voit que $b_{R}$ est dominé (uniformément en $R$ ) par une fonction sommable pour la mesure $\rho^{n-1} d \rho$. D'après le théorème de la convergence dominée, il reste à montrer que $b_{R}(\rho) \rightarrow C_{n}(\rho\|x-y\|)^{-\lambda+1 / 2} J_{\lambda-1 / 2}(\rho\|x-y\|) \cdot m(\rho)$ quand $R$ tend vers $+\infty$. La fonction $m$ étant continue à gauche, on en déduit que $m(k / R) \rightarrow m(\rho)$. Ensuite,

$$
\frac{\exp _{R} x \cdot \exp _{R} y}{R^{2}}=\exp \frac{x}{R} \cdot \exp \frac{y}{R}=\cos d\left(\exp \frac{x}{R}, \exp \frac{y}{R}\right) \text {. }
$$

Or, d'après un résultat général de géométrie riemannienne (voir [11, p. 54]), ou par un développement limité,

$$
d\left(\exp \frac{x}{R}, \exp \frac{y}{R}\right)=\frac{\|x-y\|}{R}+o\left(\frac{1}{R}\right)=\frac{\rho\|x-y\|}{k}+o\left(\frac{1}{k}\right) .
$$

D'autre part $\left(\int_{k / R}^{(k+1) / R} \rho^{n-1} d \rho\right)^{-1}=R^{n} \cdot(1+O(1 / k)) / k^{n-1}$. Le passage à la limite est achevé par une variante de la formule de Mehler-Heine (voir [22, p. 190], [23, p. 233]).

Lemme (1.2). Soit $z$ un nombre réel strictement positif; alors

$$
\lim _{k \rightarrow+\infty} \frac{1}{k^{n-1}} b Z_{k}\left(\cos \left(\frac{z}{k}+o\left(\frac{1}{k}\right)\right)\right)=C_{\lambda} \cdot z^{-\lambda+1 / 2} J_{\lambda-1 / 2}(z) .
$$


2. Etude des noyaux de Cesàro sur la sphère $\Sigma_{n}$. Rappelons d'abord la définition des sommes de Cesàro(4): on pose, pour $\operatorname{Re} \delta>-1,(1-x)^{-1-\delta}=$ $\sum_{l=0}^{+\infty} A_{l}^{\delta} x^{l}$, de sorte que

$$
A_{l}^{\delta}=\left(\begin{array}{c}
l+\delta \\
l
\end{array}\right)=\frac{(l+\delta)(l+\delta-1) \ldots(\delta+1)}{l(l-1) \cdots 1}=\frac{\Gamma(l+\delta+1)}{\Gamma(l+1) \Gamma(\delta+1)} .
$$

Si maintenant $f=\Sigma_{l=0}^{+\infty} H_{l} f$ est une décomposition orthogonale, sa Lième somme de Cesàro d'indice $\delta$ est définie par $S_{L}^{\delta} f=\left(1 / A_{L}^{\delta}\right) \Sigma_{0}^{L} A_{L-l}^{\delta} H_{l} f$. De la définition découlent immédiatement les deux égalités:

$$
A_{l+1}^{\delta}=\frac{\delta}{l+1} A_{l}^{\delta+1} \text { et } A_{L}^{\delta+\sigma+1}=\sum_{0}^{L} A_{l}^{\delta} A_{L-l}^{\sigma} .
$$

Enfin la convexité de $\log \Gamma(x)$ sur $] 0,+\infty)$ permet d'obtenir l'estimation $A_{l}^{\delta}=$ $O\left(l^{\delta}\right)(\delta \in \mathbf{R}, \delta>-1)$. Dans le cas de la sphère $\Sigma_{n}, s_{L}^{\delta} f=s_{L}^{\delta} * f$, où $s_{L}^{\delta}$ est la fonction zonale définie par $s_{L}^{\delta}=\left(1 / A_{L}^{\delta}\right) \Sigma_{0}^{L} A_{L-l}^{\delta} \mathrm{Z}_{l}$; c'est donc essentiellement le noyau de Cesàro (d'indice $\delta$ ) des développements suivant les polynômes de Gegenbauer d'indice $\lambda=(n-1) / 2$. Notons en effet que deux systèmes de pôlynomes orthogonaux de degré croissant (mais non nécessairement orthonormés) pour une même mesure ont mêmes noyaux de Cesàro.

Les noyaux de Cesàro des pôlynomes ultrasphériques, et plus généralement des polynômes de Jacobi ont été étudiés par divers auteurs ([15], [22, Chapitre IX]). Nous nous inspirons ici de la méthode de Szegö pour obtenir des estimations précises de ces noyaux, estimations qui sont indispensables pour la suite. Nous traitons le cas des polynômes de Jacobi d'indice $\alpha, \beta$ quelconques, avec toutefois la restriction que $\alpha \geq \beta \geq-1 / 2$; cela couvre en particulier l'étude des noyaux de Cesàro de tous les espaces symétriques compacts de rang 1 (voir $\$ 7$ ). Soit donc $f=\Sigma_{l=0}^{+\infty} a_{l} P_{l}^{a, \beta}$, alors

$$
S_{L}^{\delta} f(1)=\frac{1}{A_{L}^{\delta}} \sum_{l=0}^{L} A_{L-l}^{\delta} a_{l} P_{l}^{a, \beta}(1)=\int_{-1}^{+1} f(x) s_{L}^{\delta}(x)(1-x)^{a}(1+x)^{\beta} d x,
$$

$$
\text { où } s_{L}^{\delta}(x)=\left(1 / A_{L}^{\delta}\right) \Sigma_{l=0}^{L} A_{L-l}^{\delta}\left\|P_{l}^{\alpha, \beta}\right\|_{2}^{-2} P_{l}^{a, \beta}(1) P_{l}^{a, \beta}(x) \text {. }
$$

Theorème (2.1)( 5 ). Soit $\delta \geq 0$; alors

(i) si $0 \leq \theta \leq \pi / 2$,

$$
\left|S_{L}^{\delta}(\cos \theta)\right| \leq C \cdot L^{2 a+2}
$$

(ii) si $2 / L \leq \theta \leq \pi / 2$,

(4) Pour tout ce qui concerne les sommes de Cesàro, voir [25, Chapitre III].

(5) Dans le cas $a=\beta$, une partie de ces estimations a été démontrée par Kogbetliantz [15], en suivant d'ailleurs une méthode différente. 


$$
\begin{aligned}
\left|s_{L}^{\delta}(\cos \theta)\right| & \leq C \cdot L^{a+1 / 2-\delta}|\theta|^{-a-\delta-3 / 2} & & (\delta \leq a+3 / 2) ; \\
& \leq C \cdot L^{-1}|\theta|^{-2 a-3} & & (\delta \geq a+3 / 2)
\end{aligned}
$$

(iii) si $\pi / 2 \leq \theta \leq \pi-2 / L$,

$$
\begin{aligned}
\left|s_{L}^{\delta}(\cos \theta)\right| & \leq C \cdot L^{a+1 / 2-\delta}|\pi-\theta|^{-\beta-1 / 2} & & (\delta \leq \alpha+3 / 2), \\
& \leq C \cdot L^{-1}|\pi-\theta|^{-\alpha-\beta-2+\delta} & & (\alpha+3 / 2 \leq \delta \leq \alpha+\beta+2), \\
& \leq C \cdot L^{-1} & & (\delta \geq \alpha+\beta+2) ;
\end{aligned}
$$

(iv) si $\pi / 2 \leq \theta \leq \pi$,

$$
\begin{aligned}
\left|s_{L}^{\delta}(\cos \theta)\right| & \leq C \cdot L^{\alpha+\beta+1-\delta} & & (\delta \leq a+\beta+2), \\
& \leq C \cdot L^{-1} & & (\delta \geq a+\beta+2) .
\end{aligned}
$$

Avant d'entamer la démonstration, rappelons quelques estimations sur les polynômes de Jacobi:

$$
\left|P_{l}^{p, s}(\cos \theta)\right| \leq \begin{cases}C \cdot l^{p}, & 0 \leq \theta \leq \pi / 2, \\ C \cdot l^{-1 / 2} \theta^{-p-1 / 2}, & 0<\theta \leq \pi / 2, \\ C \cdot l^{-1 / 2}|\pi-\theta|^{-s-1 / 2}, & \pi / 2 \leq \theta<\pi, \\ C \cdot l^{s}, & \pi / 2 \leq \theta \leq \pi,\end{cases}
$$

(voir $[22$, p. 167])

$$
\begin{aligned}
& P_{l}^{a}, \beta(1)=\left(\begin{array}{c}
l+a \\
l
\end{array}\right)=O\left(l^{a}\right) \quad \text { (ibid., p. 58); } \\
& \left\|P_{l}^{a}, \beta_{\cdot}\right\|_{2}^{-2}=\left(\frac{2^{a+\beta+1}}{2 l+a+\beta+1} \cdot \frac{\Gamma(l+a+1) \Gamma(l+\beta+1)}{\Gamma(l+1) \Gamma(l+a+\beta+1)}\right)^{-1}=O(l) \quad \text { (ibid., p. 68). }
\end{aligned}
$$

Démonstration de (i). On utilise la majoration uniforme des polynômes de Jacobi; d'où

$$
\left|s_{L}^{\delta}\right| \leq \frac{1}{A_{L}^{\delta}} \sum_{0}^{L} A_{L-l}^{\delta} O(l) O\left(l^{a}\right) O\left(l^{a}\right) \leq c^{t e} L^{2 a+2} .
$$

Démonstration de (ii). Supposons d'abord $\delta$ entier. Szegö a montré le résultat suivant $[22$, p. 256]:

Lemme (2.2). $s_{L}^{\delta}(x)=\left(1 / A_{L}^{\delta}\right) \Sigma_{l=0}^{L} H_{l}(L, \delta) P_{l}^{a+\delta+1, \beta}(x)$, ò̀

$$
H_{l}(L, \delta) \leq \begin{cases}C \cdot L^{\delta-1} & \text { si } l=0, \\ C \sum_{\rho=0}^{\delta-1}(L-l)^{\rho} l^{a-\rho} & \text { si } 1 \leq l \leq L-1 \\ C \cdot L^{a+1} & \text { si } l=L .\end{cases}
$$


Soit d'abord $\delta<a+3 / 2$; on majore alors facilement en utilisant la deuxième majoration de $P_{l}^{a+\delta+1, \beta}$ sur $] 0, \pi / 2[$

$$
\begin{aligned}
\left|s_{L}^{\delta}(\cos \theta)\right| & \leq \frac{C}{L^{\delta}}\left(L^{\delta-1}+\sum_{l=1}^{L-1} \sum_{\rho=0}^{\delta-1}(L-l)^{\rho} l^{\alpha-\rho} l^{-1 / 2}+L^{a+1} L^{-1 / 2}\right)|\theta|^{-a-\delta-3 / 2} \\
& \leq C \cdot L^{a+1 / 2-\delta}|\theta|^{-a-\delta-3 / 2} .
\end{aligned}
$$

Si au contraire $\delta>a+3 / 2$, on majore de la même façon la contribution des termes $l=0$ et $l=L$. Ensuite on coupe en $\Sigma_{1 \leq l \leq 1 / \theta}+\Sigma_{1 / \theta \leq l \leq L / 2}+$ $\Sigma_{L / 2 \leq l \leq L-1}$, et on majore comme suit:

$$
\begin{aligned}
\sum_{L / 2}^{L-1} & \leq \frac{C}{L^{\delta}}\left(\sum_{L / 2}^{L-1} \sum_{\rho=0}^{\delta-1}(L-l)^{\rho} l^{\alpha-\rho_{l}-1 / 2}\right)|\theta|^{-\alpha-\delta-3 / 2} \\
& \leq \frac{C}{L^{\delta}}\left(\sum_{L / 2}^{L-1}(L-l)^{\delta-1}\right) L^{\alpha-\delta+1 / 2}|\theta|^{-a-\delta-3 / 2} \leq C \cdot L^{a-\delta+1 / 2}|\theta|^{-a-\delta-3 / 2},
\end{aligned}
$$

majoration qui est meilleure que celle à obtenir, compte tenu de $L \theta \geq 2$.

$$
\begin{aligned}
\sum_{1 / \theta}^{L / 2} & \leq \frac{C}{L^{\delta}}\left(\sum_{1 / \theta}^{L / 2} \sum_{\rho=0}^{\delta-1}(L-l)^{\rho} l^{\alpha-\rho} l^{-1 / 2}\right)|\theta|^{-\alpha-\delta-3 / 2} \\
& \leq \frac{C}{L^{\delta}}\left(\sum_{1 / \theta}^{L / 2} L^{\delta-1} l^{\alpha-\delta+1 / 2}\right)|\theta|^{-a-\delta-3 / 2} \leq \frac{C}{L} \cdot \theta^{-a+\delta-3 / 2-a-\delta-3 / 2} .
\end{aligned}
$$

Enfin, là où $l \leq 1 / \theta$, id est $\theta \leq 1 / l$, on utilise la majoration uniforme des polynômes de Jacobi. D'où

$$
\begin{aligned}
\sum_{1}^{1 / \theta} & \leq \frac{C}{L^{\delta}} \sum_{1}^{1 / \theta}\left(\sum_{\rho=0}^{\delta-1}(L-l)^{\rho} l^{a-\rho} l^{a+\delta+1}\right) \\
& \leq \frac{C}{L^{\delta}} L^{\delta-1} \sum_{1}^{1 / \theta} l^{2 a+2} \leq \frac{C}{L} \theta^{-2 a-3}
\end{aligned}
$$

Lorsque $\delta=a+3 / 2$ est un entier, la méthode précédente fournit une estimation un peu moins bonne que celle recherchée. Pour traiter ce cas, ainsi que les cas où $\delta$ est un indice non entier, on va utiliser une autre décomposition des noyaux de Cesàro, due elle aussi à Szegö (voir [22, p. 259]). 
Lemme (2.3).

$$
\begin{gathered}
S_{L}^{\delta}(x)=\frac{1}{A_{L}^{\delta}}\left\{2^{a+\beta+1} \frac{\Gamma(\alpha+1) \Gamma(L+\beta+1) \Gamma(2 L+\alpha+\beta+2 \delta+3)}{\Gamma(L+\alpha+\beta+\delta+2) \Gamma(2 L+\alpha+\beta+\delta+3)}\right\}^{-1} P_{l}^{\alpha+\delta+1, \beta}(x) \\
-\sum_{\rho=1}^{+\infty}(-1)^{\rho} \frac{(L+\delta+1) \ldots(L+\delta+\rho)}{(2 L+\alpha+\beta+\delta+3) \ldots(2 L+\alpha+\beta+\delta+\rho+2)} \\
\frac{\delta(\delta-1) \ldots(\delta-\rho+1)}{1 \cdot 2 \ldots \ldots \rho} s_{L}^{\delta+\rho}(x) .
\end{gathered}
$$

Avant d'utiliser ce lemme, montrons un résultat auxiliaire.

Lemme (2.4). Soit $\delta_{0}$ un entier, $\delta_{0} \neq \alpha+3 / 2 ;$ si $\delta>\delta_{0}$, et désignant par $\tau_{L}^{\delta_{0}}(\theta)$ la fonction majorante qui figure dans le Théorème (2.1), on a $\left|s_{L}^{\delta}(\cos \theta)\right| \leq$ $C_{\delta_{0}} \cdot \delta \cdot \tau_{L}^{\delta_{0}}(\theta)$.

On a en effet la relation $s_{L}^{\delta}=\left(1 / A_{L}^{s}\right) \Sigma_{l=0}^{L} A_{L-l}^{\delta-\delta_{0}-1} A_{l}^{\delta_{0}} s_{l}^{\delta_{0}}$; majorant $\left|s_{l}^{\delta_{0}}\right|$ par $\tau_{l}^{\delta_{0}}$, et utilisant la forme particulière de cette majorante sur les divers intervalles on voit qu'il suffit de démontrer l'inégalité $\left(1 / A_{L}^{\delta}\right) \Sigma_{l=0}^{L} A_{L-l}^{\delta-\delta_{0}-1} A_{l}^{\delta_{0}}(l+1)^{\gamma_{0}}$ $\leq C_{\delta_{0}} \cdot \delta \cdot(L+1)^{\gamma_{0}}$, où $\gamma_{0} \geq-1$. Noter qu'on a changé $l$ en $(l+1)$ dans les estimations, ce qui est toujours possible, et indispensable quand $l=0$. On écrit alors $(l+1)^{\gamma_{0}}=(l+1)^{-1}(l+1)^{\gamma_{0+1}} \leq L^{\gamma_{0}} \cdot L \cdot(l+1)^{-1}$ de sorte qu'il suffit de montrer le résultat lorsque $\gamma_{0}=-1$. Or $A_{l}^{\delta_{0}} /(l+1)=A_{l+1}^{\delta_{0}-1} / \delta_{0}$, de sorte que la somme à majorer s'écrit:

$$
\frac{1}{A_{L}^{\delta}} \sum_{l=0}^{L} A_{L+1-(l+1)}^{\delta-\delta_{0}-1} A_{l+1}^{\delta_{0}^{-1}}=\frac{1}{A_{L}^{\delta}} \cdot A_{L+1}^{\delta-1}=\frac{\delta}{L+1} .
$$

Cela étant, revenons à la formule de Szegö. Le coefficient du premier terme est $O\left(L^{a+1-\delta}\right)$, et par suite ce premier terme se majore dans tous les cas comme il faut. La sommation ne fait intervenir que des indices supérieurs ou égaux au premier entier strictement plus grand que $\delta$ (dans le cas où $\delta=\alpha+3 / 2$ est entier, le premier indice qui intervient est $\alpha+5 / 2$ ), de sorte qu'on peut majorer $\left|s_{L}^{\delta+\rho}\right|$ par $C \cdot(\rho+\delta) \cdot \tau_{L}^{[\delta]+1}(\theta)$. Concernant le coefficient, on note d'abord que $|(\delta(\delta-1) \cdots(\delta-\rho+1)) /(1 \cdots \rho)| \leq C \cdot \rho^{-1-\delta}$; ensuite

$$
\begin{aligned}
& \frac{(L+\delta+1) \cdots(L+\delta+\rho)}{(2 L+\alpha+\beta+\delta+3)} \cdots(2 L+\alpha+\beta+\delta+\rho+2) \\
& \leq C \cdot \frac{(\delta+2) \ldots(\delta+\rho+1)}{(\alpha+\beta+\delta+5) \cdots(\alpha+\beta+\delta+\rho+4)},
\end{aligned}
$$

car la fonction $(x+\delta+k) /(2 x+\alpha+\beta+\delta+k+1)$ est décroissante (dès lors que $k>(\alpha+\beta+1)-\delta)$. Ce dernier coefficient est donc $O\left(\rho^{-(\alpha+\beta+3)}\right)$. D'oú finalement 


$$
\sum_{\rho=1}^{+\infty} \leq C\left(\sum_{\rho=1}^{+\infty} \rho^{1-\delta} \cdot \rho^{-(\alpha+\beta+3)} \cdot \rho\right) \tau_{L}^{[\delta]+1}(\theta) \leq C \cdot \tau_{L}^{[\delta]+1}(\theta) .
$$

On utilise d'abord ce résultat pour obtenir le cas où $\delta=\alpha+3 / 2$ est entier, puis le cas général.

Les majorations de (iii) et (iv) sont fondées sur les mêmes lemmes et ne comportent pas de difficultés nouvelles.

Corollaire (2.5). Soit $s_{L}^{\delta}$ le noyau de Cesàro d'indice $\delta$ de $\Sigma_{n}$; alors

(i) si $d(x, 1) \leq \pi / 2$,

$$
\left|s_{L}^{\delta}(x)\right| \leq C \cdot L^{n}
$$

(ii) si $2 / L \leq d(x, 1) \leq \pi / 2$,

$$
\begin{aligned}
\left|s_{L}^{\delta}(x)\right| & \leq C \cdot L^{((n-1) / 2-\delta)} d(x, 1)^{-(n-1) / 2-\delta-1} & & (\delta \leq(n+1) / 2), \\
& \leq C \cdot L^{-1} d(x, 1)^{-n-1} & & (\delta \geq(n+1) / 2)
\end{aligned}
$$

(iii) si $\pi / 2 \leq d(x, 1) \leq \pi-2 / L$,

$$
\begin{aligned}
\left|s_{L}^{\delta}(x)\right| & \leq C \cdot L^{((n-1) / 2-\delta)} d(x,-1)^{-(n-1) / 2} & & (\delta \leq(n+1) / 2), \\
& \leq C \cdot L^{-1} d(x,-1)^{-n+\delta} & & ((n+1) / 2 \leq \delta \leq n), \\
& \leq C \cdot L^{-1} & & (\delta \geq n) ;
\end{aligned}
$$

(iv) si $\pi-2 / L \leq d(x, 1) \leq \pi$,

$$
\begin{aligned}
\left|s_{L}^{\delta}(x)\right| & \leq C \cdot L^{n-1-\delta} & & (\delta \leq n), \\
& \leq C \cdot L^{-1} & & (\delta \geq n) .
\end{aligned}
$$

Remarques. (1) Signalons que E. Kogbetliantz a montré dans [15] que si $\delta \geq n$, alors $s_{L}^{\delta} \geq 0$ en tout point; c'est une généralisation du résultat classique de Fejér (cas $n=1$ ). Par suite, on doit s'attendre à ce que les majorations de $s_{L}^{\delta}$ ne s'améliorent plus lorsque $\delta$ devient supérieur à $n$.

(2) Le noyau de Poisson $P_{r}(x)=\Sigma_{0}^{+\infty} r^{k} Z_{k}(x)=\left(1-r^{2}\right) /|r x-1|^{n+1}$ satisfait les majorations suivantes:

$$
\begin{aligned}
P_{r}(x) & \leq C \cdot(1-r)^{-n}, & & 0 \leq d(x, 1) \leq \pi, \\
& \leq C \cdot d(x, 1)^{-n-1} \cdot(1-r), & & 0 \leq d(x, 1) \leq \pi / 2, \\
& \leq C \cdot(1-r), & & \pi / 2 \leq d(x, 1) \leq \pi,
\end{aligned}
$$

et le calcul de $P_{r}$ en $1,-1$ et un point équatorial montre qu'on ne peut espérer améliorer ces majorations. Or le noyau de Poisson s'exprime en fonction des sommes de Cesàro d'indice $\delta$ par la formule $P_{r}=(1-r)^{1+\delta} \Sigma_{l=0}^{+\infty} A_{l}^{\delta} r^{l} s_{l}^{\delta}$. Substituant alors à $r$ la quantité $1-1 / L$ (cf. la remarque de [25, p. 223]), on voit que 
toute majoration de $s_{L}^{\delta}$ en $l^{-\alpha} \theta^{-\beta}$ se transfère immédiatement au noyau $P_{1-1 / L^{\circ}}$. Les paliers observés dans les majorations $(\delta=(n+1) / 2$, et $\delta=n)$ sont bien ceux que laissent prévoir cette remarque.

(3) Les remarques précédentes valent encore pour les polynômes ultrasphériques d'indice $\lambda$ quelconque. Dans le cas des polynômes de Jacobi quelconques, Askey a conjecturé que $s_{L}^{\delta} \geq 0$, dès lors que $\delta \geq a+\beta+2$.

3. Résultats de convergence des développements en harmoniques sphériques. Il était bien connu que les fonctions $s_{L}^{\delta}$ appartiennent uniformément à $L^{1}\left(\Sigma_{n}\right)$, dès que $\delta>(n-1) / 2$, et donc que les moyennes de Cesàro d'ordre $\delta>(n-1) / 2$ d'une fonction $f$ de $L^{p}\left(\Sigma_{n}\right)$ convergent dans $L^{p}\left(\Sigma_{n}\right)$ vers $f(1 \leq p<+\infty)$. Au $\$ 5$, nous verrons comment ce résultat peut être amélioré lorsque $p>1$ et $\delta<(n-1) / 2$.

Nous allons, dans ce paragraphe, nous intéresser à d'autres types de convergence: les majorations du $\$ 2$ vont nous permettre d'obtenir des résultats de localisation et de convergence presque partout. Notre premier souci va être d'interpréter ces majorations.

$$
\begin{aligned}
\text { Posons } s_{L}^{\delta}(\cos \theta) & =\sigma_{L}^{\delta}(\cos \theta)+\tau_{L}^{\delta}(\cos \theta), \text { où } \\
& \sigma_{L}^{\delta}(\cos \theta)=s_{L}^{\delta}(\cos \theta) \times[0, \pi / 2]
\end{aligned}
$$

et de même $S_{L}^{\delta} f=\sigma_{L}^{\delta} f+\tau_{L}^{\delta} f$.

Si $\lambda=(n-1) / 2 \geq \delta$, il résulte de la majoration (iii) du théorème que:

Soit $\delta<2 \lambda$ :

$$
\begin{array}{ll}
\left|\tau_{L}^{\delta}(\cos \theta)\right| \leq A|\pi-\theta|^{-2 \lambda+\delta} & \text { si } \delta \leq 2 \lambda, \\
\left|\tau_{L}^{\delta}(\cos \theta)\right| \leq A & \text { si } \delta \geq 2 \lambda .
\end{array}
$$

$$
\left\|\sup _{L}\left|\tau_{L}^{\delta}\right|\right\|_{p} \leq A\left(\int_{\pi / 2}^{\pi}|\pi-\theta|^{(-2 \lambda+\delta) p}|\pi-\theta|^{2 \lambda} d \theta\right)^{1 / p}<\infty \text { si } p<\frac{2 \lambda+1}{2 \lambda-\delta} .
$$

Donc $\sup _{L}\left|\tau_{L}^{\delta}\right| \in L^{p}\left(\Sigma_{n}\right)$ pour $p<n /(n-1-\delta)$ si $(n-1) / 2 \leq \delta<n-1$, $\sup _{L}\left|\tau_{L}^{\delta}\right| \in L^{\infty}\left(\Sigma_{n}\right)$ si $\delta \geq n-1$.

Montrons d'autre part que, si $\delta>\lambda=(n-1) / 2$, il existe une constante $A$ telle que, pour tout $f \in L^{1}\left(\Sigma_{n}\right)$ et pour tout $x \in \mathbf{R}^{n}, \sup _{L}\left|\sigma_{L}^{\delta} f(x)\right| \leq A M f(x)(6)$. Quitte à changer $f$, on peut supposer que $x=1$.

$$
\begin{aligned}
\sigma_{L}^{\delta} f(1) & =\int_{\Sigma_{n}} \sigma_{L}^{\delta}(x \cdot 1) f(x) d_{i}^{l} \sigma(x) \\
& =\int_{0}^{\pi} \sigma_{L}^{\delta}(\cos \theta) \int_{\Sigma_{n-1}} f(\sin \theta \xi, \cos \theta) \sin ^{n-1} \theta d \xi d \theta
\end{aligned}
$$

(6) On désigne par $M f$ la fonction maximale de Hardy-Littlewood (cf. introduction) $M f(x)=\sup _{r>0}\left(\int_{d(x, y) \leq r}|f(y)| d \sigma(y)\right) / \int_{d(x, y) \leq r} i \sigma(y)$. 
Posons:

$$
\begin{aligned}
& \Lambda(\theta)=\int_{x \cdot 1 \geq \cos \theta}|f(x)| d \sigma(x)=\int_{0}^{\theta} \int_{\Sigma_{n-1}}|f(\sin \phi \xi, \cos \phi)| d \xi \sin ^{n-1} \phi d \phi, \\
& \Lambda(\theta) \leq M f(1) \int_{x \cdot 1 \geq \cos \theta} d \sigma(x)=C_{n} M f(1) \int_{0}^{\theta} \sin ^{n-1} \phi d \phi .
\end{aligned}
$$

Posons d'autre part:

$$
\begin{array}{ll}
k_{L}(\theta)=L^{\lambda-\delta}\left(L^{-2}+\theta^{2}\right)^{(-\lambda-\delta-1) / 2} & \text { si } \delta \leq \lambda+1, \\
k_{L}(\theta)=L^{-1}\left(L^{-2}+\theta^{2}\right)^{-\lambda-1} & \text { si } \delta \geq \lambda+1 .
\end{array}
$$

En vertu du Corollaire (2.5), $\sigma_{L}^{\delta}(\cos \theta) \leq A k_{L}(\theta)$,

$$
\left|\sigma_{L}^{\delta} f(1)\right| \leq A\left[\int_{0}^{\pi}\left(-k_{L}^{\prime}(\theta)\right) \Lambda(\theta) d \theta+\Lambda(\pi) k_{L}(\pi)\right]
$$

comme le montre une intégration par parties et le fait que $k_{L}^{\prime}$ est négatif

$$
\left|\sigma_{L}^{\delta} f(1)\right| \leq A\left(-\int_{0}^{\pi} k_{L}^{\prime}(\theta) \int_{0}^{\theta} \sin ^{n-1} \phi d \phi d \theta+k_{L}(\pi) \int_{0}^{\pi} \sin ^{n-1} \phi d \phi\right) M f(1) .
$$

Or

$$
-\int_{0}^{\pi} k_{L}^{\prime}(\theta) \int_{0}^{\theta} \sin ^{n-1} \phi d \phi=-k_{L}(\pi) \int_{0}^{\pi} \sin ^{n-1} \phi d \phi+\int_{0}^{\pi} k_{L}(\theta) \sin ^{n-1} \theta d \theta .
$$

Comme $\sup _{L} \int_{0}^{\pi} k_{L}(\theta) \sin ^{n-1} \theta d \theta<\infty$, il existe une constante $A$ telle que $\sigma_{L}^{\delta} f(1) \leq A M f(1)$.

On peut résumer ces résultats dans le théorème suivant:

Théorème (3.1). Soit $\delta>(n-1) / 2$. Il existe une constante $A$ indépendante de la fonction $f \in L^{1}\left(\Sigma_{n}\right)$ telle que $\sup _{L}\left|S_{L}^{\delta} f\right| \leq A(M f+K *|f|)$ où $K$ est une fonction positive qui appartient à $L^{p}\left(\Sigma_{n}\right)$ pour les valeurs de p inférieures à $n /(n-1-\delta)$ lorsque $\delta<n-1$, ’a $L^{\infty}\left(\Sigma_{n}\right)$ lorsque $\delta \geq n-1$.

Il est à remarquer que la majoration $\sup _{L}\left|S_{L}^{\delta} f\right| \leq A M f$ lorsque $\delta \geq n-1$ a été obtenue dans un cadre beaucoup plus général par Hörmander ([12], [13]), du moins lorsque les moyennes de Cesàro sont remplacées par les moyennes de Riesz. Nos résultats vont nous permettre, dans ce cas particulier, d'obtenir des théorèmes de localisation plus précis.

Théorème (3.2). Soit $\epsilon>0, \delta \geq(n-1) / 2$. Si la fonction $f$ appartient à l'espace $L^{p}\left(\Sigma_{n}\right), p \geq 1$, où $p>n /(\delta+1)$, et si f s'annule à l'intérieur de la boule $d(x, 1)<\epsilon$, alors $S_{L}^{\delta} f(1)$ tend vers 0 , lorsque $L$ tend vers $l$ 'infini.

En utilisant la densité des fonctions $C^{\infty}$ dans $L^{p}\left(\Sigma_{n}\right)$, on se ramène à montrer l'existence d'une constante $A_{p}$ indépendante de $f$ telle $\sup _{L}\left|S_{L}^{\delta} f(1)\right| \leq$ $A_{p}\|f\|_{p}$ pour toute fonction $f$ s'annulant à l'intérieur de la boule $d(x, 1)<\epsilon$. 
Cette dernière inégalité est satisfaite si $s_{L}^{\delta}(\cos \theta) \chi_{[\epsilon, \pi]}(\theta)$ appartient uniformément à $L^{p^{\prime}}\left(\sin ^{2 \lambda} \theta d \theta\right), 1 / p+1 / p^{\prime}=1$. Or, si $\epsilon \geq L^{-1}$,

$$
\begin{gathered}
\left|\sigma_{L}^{\delta}(\cos \theta)\right| \leq A L^{\lambda-\delta} \theta^{-\lambda-\delta-1} \leq A \epsilon^{-\lambda-\delta-1} \quad \text { lorsque } \theta \geq \epsilon ; \\
\left\|\tau_{L}^{\delta}\right\|_{p^{\prime}} \leq A \text { si } p>n /(\delta+1) .
\end{gathered}
$$

Remarque. On peut montrer que les conditions du Théorème (3.2) sont des conditions nécessaires pour qu'il y ait localisation. Supposons tout d'abord $\delta>(n-1) / 2$, et $p<n /(\delta+1)$ : la fonction $(1+x \cdot 1)^{-\mu}$, qui appartient à $L^{p}\left(\Sigma_{n}\right)$ lorsque $p<n / 2 \mu$, est $\mathcal{C}^{\infty}$ ou voisinage de 1 . Il existe donc une fonction qui est $C^{\infty}$ sur $\Sigma_{n}$, dont les moyennes de Cesàro $S_{L}^{\delta} f(1)$ convergent vers $f(1)$ de ce fait, et qui coincide avec $(1+x \cdot 1)^{-\mu}$ dans la boule $d(x, 1)<\epsilon$. Comme les moyennes de Cesàro d'ordre $2 \mu-1$ de $(1+x \cdot 1)^{-\mu}$ ne convergent pas en 1 (voir $[22$, p. 265]), on obtient ainsi un contre-exemple explicite avec $\delta=2 \mu-1$.

Plus généralement, en vertu du théorème de Banach-Steinhaus, les conclusions du Théorème (3.2) ont lieu si et seulement si $\sup _{L}\left|S_{L}^{\delta} f(1)\right| \leq A\|f\|_{p}$ quelle que soit la fonction $f \in L^{p}\left(\Sigma_{n}\right)$ nulle dans la boule $d(x, 1)<\epsilon$, et donc si et seulement si $\left.s_{L}^{\delta}(\cos \theta) \chi[\epsilon, \pi]\right]^{(\theta)}$ appartient uniformément à $L^{p^{\prime}}\left(\sin ^{2 \lambda} \theta d \theta\right)$.

Soit alors $(n-1) / 2 \leq \delta<n-1, p=n /(\delta+1)$. On veut montrer que $\tau_{L}^{\delta}(\cos \theta)$ n'appartient pas uniformément à $L^{p^{\prime}}\left(\sin ^{2 \lambda} \theta d \theta\right)$. En utilisant le Lemme (2.3), on voit aisément qu'il suffit de montrer que $L^{\lambda-\delta+1 / 2} P_{L}^{\lambda+\delta+1 / 2, \lambda+1 / 2}(\cos \theta) \chi_{[\pi / 2, \pi]}(\theta)$ n'appartient pas uniformément à $L^{p^{\prime}}\left(\sin ^{2 \lambda} \theta d \theta\right)$. Cette dernière propriété découle de la formule asymptotique de Mehler-Heine, comme on peut le voir en utilisant la méthode de Szegö $[22$, p. 173].

De même, lorsque $\delta<(n-1) / 2=\lambda$, on est ramené à montrer que $L^{\lambda-\delta+1 / 2} P_{L}^{\lambda+\delta+1 / 2, \lambda+1 / 2}(\cos \theta) \chi[\epsilon, \pi / 2]^{(\theta)}$ n'appartient pas uniformément à $L^{1}\left(\sin ^{2 \lambda} \theta d \theta\right)$ pour prouver que, quel que soit $p$, il n'y a pas localisation pour les fonctions de $L^{p}\left(\Sigma_{n}\right)$. Or

$$
\int_{\epsilon}^{\pi / 2} \sin ^{2 \lambda} \theta\left|P_{L}^{\lambda+\delta+1 / 2, \lambda+1 / 2}(\cos \theta)\right| d \theta \geq A_{\mu} \int_{\epsilon}^{\pi / 2} \sin ^{2 \mu} \theta\left|P_{L}^{\lambda+\delta+1 / 2, \lambda+1 / 2}(\theta)\right| d \theta
$$

quel que soit $\mu$. Si $\mu$ est suffisamment grand, cette dernière quantité est minorée par $L^{-1 / 2}[22$, p. 173].

Théorème (3.3). Soit $\delta>(n-1) / 2$. Il existe une constante A telle que, pour toute fonction $\left.f \in L^{1}\left(\Sigma_{n}\right), \operatorname{msup}_{L}\left|S_{L}^{\delta} f\right|>\alpha\right\} \leq(A / \alpha)\|f\|_{1}$. Les moyennes de Ces'aro d'ordre $\delta$ d'une fonction de $L^{1}\left(\Sigma_{n}\right)$ convergent presque partout.

La convergence presque partout est une conséquence de l'inégalité faible, qui découle du Théorème (3.1).

Corollaire (3.4). Soit $1<p \leq 2$. Si $\delta>(n-1)[1 / p-1 / 2]$, il existe une 
constante $A_{p}$ telle que, pour tout $f \in L^{p}\left(\Sigma_{n}\right),\left\|\sup _{L}\left|S_{L}^{\delta} f\right|\right\|_{p} \leq A_{p}\|f\|_{p}$. Les moyennes de Cesàro d'ordre $\delta$ d'une fonction de $L^{p}\left(\Sigma_{n}\right)$ convergent presque partout.

Le résultat, lorsque $p=2$, découle du lemme suivant, qui est un cas particulier d'un résultat général sur les systèmes orthonormaux:

Lemme (3.5). Si, pour une valeur $\delta_{0}>0$, les moyennes d'ordre $\delta_{0}$ des fonctions de $L^{2}\left(\Sigma_{n}\right)$ satisfont à l'inégalité $\left\|\sup _{L}\left|S_{L}^{\delta_{0}} f\right|\right\|_{2} \leq A_{\delta_{0}}\|f\|_{2}$, alors, quel que soit $\delta>0$, elles satisfont à l'inégalité $\left\|\sup _{L}\left|S_{L}^{\delta} f\right|\right\|_{2} \leq A_{\delta}\|f\|_{2}$.

La démonstration, dans le cas de $\mathbf{R}^{n}$ et lorsque les moyennes de Cesàro sont remplacées par les moyennes de Riesz, se trouve dans [12]. Elle est tout à fait analogue dans le cas présent. Nous en donnerons seulement un aperçu.

Les moyennes de Cesàro d'ordre $\delta+\beta$ s'obtiennent à partir des moyennes de Cesàro d'ordre $\delta$ par la formule

$$
\begin{aligned}
S_{L}^{\delta+\beta} f & =\left(A_{L}^{\delta+\beta}\right)^{-1} \sum_{k=0}^{L} A_{k}^{\delta} A_{L-k}^{\beta-1} S_{k}^{\delta} f . \\
\left(A_{L}^{\delta+\beta}\right)^{-2} \sum_{k=0}^{L}\left(A_{k}^{\delta} A_{L-k}^{\beta-1}\right)^{2} & =O\left(L^{-2 \delta-2 \beta} \sum_{k=0}^{L} k^{2 \delta}(L-k)^{2 \beta-2}\right) \\
& =O\left(L^{-1}\right) \quad \text { si } \beta>1 / 2 .
\end{aligned}
$$

Donc, en vertu de l'inégalité de Schwarz, il suffit de montrer que

$$
\left\|\sup _{L}\left(\frac{1}{L} \sum_{k=0}^{L}\left|S_{k}^{\delta} f\right|^{2}\right)^{1 / 2}\right\|_{2} \leq A_{\delta}\|f\|_{2}
$$

pour tout $\delta>-1 / 2$.

Or, par hypothèse,

$$
\left\|\sup _{L}\left(\frac{1}{L} \sum_{k=0}^{L}\left|S_{k}^{\delta_{0}} f\right|^{2}\right)^{1 / 2}\right\|_{2} \leq\left\|\sup _{L} \mid S_{L}^{\delta} 0 f\right\|_{2} \leq A_{\delta_{0}}\|f\|_{2} .
$$

Il suffit donc, pour prouver le résultat, de démontrer que, si $\delta>-1 / 2$, $\left\|\left(\Sigma_{L=0}^{\infty}\left|S_{L}^{\delta+1} f-S_{L}^{-\delta} f\right|^{2} L^{-1}\right)^{1 / 2}\right\|_{2} \leq A_{\delta}\|f\|_{2}$. Posons

$$
g_{\delta}(f)=\left(\sum_{L=0}^{\infty}\left|S_{L}^{\delta+1} f-S_{L}^{\delta} f\right|^{2} L^{-1}\right)^{1 / 2}
$$




$$
\begin{aligned}
S_{L}^{\delta+1} f-S_{L}^{\delta} f & =\sum_{k=0}^{L}\left(\frac{A_{L-k}^{\delta+1}}{A_{L}^{\delta+1}}-\frac{A_{L-k}^{\delta}}{A_{L}^{\delta}}\right) H_{k} f=\frac{1}{\delta+1}\left(A_{L}^{\delta+1}\right)^{-1} \sum_{k=0}^{L} k A_{L-k}^{\delta} H_{k} f, \\
\left\|g_{\delta}(f)\right\|_{2}^{2} & =\frac{1}{(\delta+1)^{2}} \sum_{L=0}^{\infty} L^{-1}\left(A_{L}^{\delta+1}\right)^{-2} \sum_{k=0}^{L} k^{2}\left(A_{L-k}^{\delta}\right)^{2}\left\|H_{k} f\right\|_{2}^{2} \\
& =\frac{1}{(\delta+1)^{2}} \sum_{k=0}^{\infty} k^{2}\left\|H_{k} f\right\|_{2}^{2}\left(\sum_{L=k}^{\infty} L^{-1}\left(A_{L}^{\delta+1}\right)^{-2}\left(A_{L-k}^{\delta}\right)^{2}\right) \leq A_{\delta}\|f\|_{2}^{2}
\end{aligned}
$$

puisque $\Sigma_{k}^{\infty} L^{-1}\left(A_{L}^{\delta+1}\right)^{-2}\left(A_{L-k}^{\delta}\right)^{2}=O\left(\Sigma_{k}^{\infty} L^{-1} L^{-(2 \delta+2)}(L-k)^{2 \delta}\right)=O\left(k^{-2}\right)$.

Reprenons la démonstration du Corollaire (3:4): il suffit d'interpoler entre le résultat $p_{0}, p_{0}>1$, et le résultat $L^{2}$ en utilisant la méthode de Stein sur les familles analytiques d'opérateurs [18], $S_{L}^{\delta}$ étant défini pour des valeurs complexes de $\delta$.

$$
S_{L}^{\delta+\epsilon+i y} f=\left(A_{L}^{\delta+\epsilon+i y}\right)^{-1} \sum_{k=0}^{L} A_{k}^{\delta} A_{L-k}^{\epsilon-1+i y} S_{k}^{\delta} f,
$$

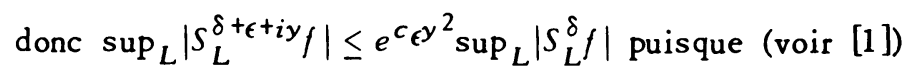

$$
\sup _{L}\left|A_{L}^{\delta+\epsilon+i y}\right|^{-1} \sum_{k=0}^{L} A_{k}^{\delta}\left|A_{L-k}^{\epsilon-1+i y}\right| \leq e^{c \epsilon y^{2}}
$$

On en déduit que si $\delta>(n-1) / 2$ et $p_{0}>1$,

$$
\left\|\sup _{L}\left|S_{L}^{\delta+i y} f\right|\right\|_{p_{0}} \leq A_{p_{0}} e^{c y^{2}}\|f\|_{p_{0}}
$$

si $\delta>0$,

$$
\left\|\sup _{L}\left|S_{L}^{\delta+i y} f\right|\right\|_{2} \leq A_{2} e^{c y^{2}}\|f\|_{2} .
$$

Nous allons terminer ce paragraphe en montrant l'inégalité suivante pour les moyennes de Cesàro, inégalité qui est l'outil fondamental pour obtenir les théorèmes de multiplicateurs:

Théorème (3.6). Soit $p>1$ et $\delta \geq(n-1) / 2$. Il existe une constante $A_{p}$ telle que pour toute suite de fonctions $f_{k}$ et toute suite d'entiers $L_{k}$ '

$$
\left\|\left(\sum\left|S_{L_{k}}^{\delta} f_{k}\right|^{2}\right)^{1 / 2}\right\|_{p} \leq A_{p}\left\|\left(\sum\left|f_{k}\right|^{2}\right)^{1 / 2}\right\|_{p} .
$$

Supposons tout d'abord $\delta>(n-1) / 2$, cette inégalité découle, en vertu du Théorème (3.1), des deux inégalités 


$$
\left\|\left(\sum\left|M f_{k}\right|^{2}\right)^{1 / 2}\right\|_{p} \leq A_{p}\left\|\left(\sum\left|f_{k}\right|^{2}\right)^{1 / 2}\right\|_{p},
$$

inégalité de Fefferman-Stein [8] et

$$
\left\|\left(\sum\left(K *\left|f_{k}\right|\right)^{2}\right)^{1 / 2}\right\|_{p} \leq A_{p}\left\|\left(\sum\left|f_{k}\right|^{2}\right)^{1 / 2}\right\|_{p},
$$

qui est une inégalité bien connue due à Marcinkiewicz et Zygmund ([25, tome 2, p. 224]).

Le cas $\delta=(n-1) / 2$ est un cas particulier du corollaire suivant:

Corollaire (3.7). Soit $1<p \leq 2$. Si $\delta>(n-1)[1 / p-1 / 2]$, les moyennes de Cesàro d'ordre $\delta$ satisfont à l' inégalité $\left\|\left(\Sigma\left|S_{L_{k}}^{\delta} f_{k}\right|^{2}\right)^{1 / 2}\right\|_{p} \leq A_{p}\left\|\left(\Sigma\left|f_{k}\right|^{2}\right)^{1 / 2}\right\|_{p}$ quelles que soient les suites $f_{k}$ et $L_{k}$.

Remarquons que

$$
\left|S_{L_{k}}^{\delta+\epsilon+i y} f_{k}\right|^{2} \leq e^{c \epsilon^{y^{2}}}\left(\left|A_{L_{k}}^{\delta+\epsilon+i y}\right|^{-1}\right) \sum_{j=0}^{L_{k}} A_{j}^{\delta}\left|A_{L_{k}}^{\epsilon-1+i y}\right|\left|\delta_{j}^{\delta} f_{k}\right|^{2}
$$

en vertu de l'inégalité de Schwarz.

Si l'inégalité $\left\|\left(\Sigma_{k}\left|S_{L_{k}}^{\delta} f_{k}\right|^{2}\right)^{1 / 2}\right\|_{p} \leq A_{p}\left\|\left(\Sigma_{k}\left|f_{k}\right|^{2}\right)^{1 / 2}\right\|_{p}$ est satisfaite pour toutes les suites $L_{k}$ et $f_{k}$, alors, en particulier,

$$
\begin{gathered}
\left\|\left(\sum_{k}\left|A_{L_{k}}^{\delta+\epsilon+i y}\right|^{-1} \sum_{j=0}^{L_{k}} A_{j}^{\delta}\left|A_{L_{k}}^{\epsilon-1+i y}\right|\left|S_{j}^{\delta} f_{k}\right|^{2}\right)^{1 / 2}\right\|_{p} \\
\leq A_{p}\left\|\left(\sum_{k}\left|A_{L_{k}+\epsilon+i y}\right|^{-1} \sum_{j=0}^{L_{k}} A_{j}^{\delta}\left|A_{L_{k}}^{\epsilon-1+i y}\right|\left|f_{k}\right|^{2}\right)^{1 / 2}\right\|_{p} \\
\leq A_{p} e^{c \epsilon^{y^{2} / 2}}\left\|\left(\sum_{k}\left|f_{k}\right|^{2}\right)^{1 / 2}\right\|_{p},
\end{gathered}
$$

donc $\left\|\left(\Sigma_{k}\left|S_{L_{k}}^{\delta+\epsilon+i y} f_{k}\right|^{2}\right)^{1 / 2}\right\|_{p} \leq A_{p} e^{c \epsilon y^{2}}\left\|\left(\Sigma\left|f_{k}\right|^{2}\right)^{1 / 2}\right\|_{p}$. En particulier, si $\delta>$ $(n-1) / 2$ et $p>1$, ou bien si $\delta>0$ et $p=2$,

$$
\left\|\left(\sum_{k}\left|S_{L_{k}}^{\delta+i y} f_{k}\right|^{2}\right)^{1 / 2}\right\|_{p} \leq A_{p} e^{c y^{2}}\left\|\left(\sum\left|f_{k}\right|^{2}\right)^{1 / 2}\right\|_{p} .
$$

Le Corollaire (3.7) s'en déduit par interpolation.

4. Thérèmes de multiplicateurs. Soit $f \in L^{1}\left(\Sigma_{n}\right)$. On note $P_{r} f$ la fonction

$$
P_{r} f=\sum r^{k} H_{k} f, \quad 0<r<1 .
$$


$P_{r} f$ est la convolée de la fonction $f$ avec le "noyau de Poisson" sur $\Sigma_{n}$ défini par

$$
P_{r}(x)=\sum_{0}^{\infty} r^{k} Z_{1}^{k}(x)=\frac{1-r^{2}}{|r x-1|^{n+1}} .
$$

$P_{r}$ est une fonction positive, uniformément dans $L^{1}\left(\Sigma_{n}\right)$. Soit $g(f)$ la fonction de Littlewood-Paley définie par

$$
g(f)=\left(\int_{0}^{1}(1-r)\left|\frac{\partial}{\partial r}\left(P_{r} f\right)\right|^{2} d r\right)^{1 / 2} .
$$

Il existe, si $p>1$, une constante $A_{p}$ telle que $\|g(f)\|_{p} \leq A_{p}\|f\|_{p}$ et, si $H_{0} f=$ $\int_{\Sigma_{n}} f d x=0,\|f\|_{p} \leq A_{p}\|g(f)\|_{p}$ (voir [19, Chapitres 3 et 4], [5, p. 149]) (7).

Les théorèmes de multiplicateurs découleront de l'étude des fonctions de Littlewood-Paley modifiées suivantes: $g_{\delta}(f)=\left(\Sigma_{L}\left|S_{L}^{\delta+1} f-S_{L}^{\delta} f\right|^{2} L^{-1}\right)^{1 / 2}$ pour lesquelles on a le théorème suivant:

Théorème (4.1). Soient $p>1$ et $\delta \geq 0$ tels que, pour toute suite de fonctions $f_{k}$ et toute suite d'entiers $L_{k},\left\|\left(\Sigma\left|S_{L_{k}}^{\delta} f_{k}\right|^{2}\right)^{1 / 2}\right\|_{p} \leq A_{p}\left\|\left(\Sigma\left|f_{k}\right|^{2}\right)^{1 / 2}\right\|_{p}$. Alors, pour toute fonction $f \in L^{p}\left(\Sigma_{n}\right)$, $\left\|_{\delta}(f)\right\|_{p} \leq A_{p}\|f\|_{p}$ et, si $H_{0} f=0,\|f\|_{p} \leq A_{p}\left\|_{g \delta}(f)\right\|_{p}$.

La seconde inégalité est vraie sans restriction sur $\delta$ puisqu'elle provient de l'inégalité ponctuelle suivante:

Lemme (4.2). $g(f) \leq C \cdot g_{\delta}(f)$.

En effet,

$$
\begin{aligned}
& \frac{\partial}{\partial r} P_{r} f=\sum_{k=1}^{\infty} k r^{k-1} H_{k} f ; \\
& S_{L}^{\delta+1} f-S_{L}^{\delta} f \sim L^{-1}\left(A_{L}^{\delta}\right)^{-1} \sum_{k=0}^{L} k A_{L-k}^{\delta} H_{k} f, \\
& \frac{\partial}{\partial r} P_{r} f=(1-r)^{\delta+1} \sum_{L=1}^{\infty}\left(\sum_{k=0}^{L} k A_{L-k}^{\delta} H_{k} f\right) r^{L-1} \\
& \quad\left(\text { puisque } \sum_{k=0}^{\infty} A_{k}^{\delta} r^{k}=(1-r)^{-\delta-1}\right)
\end{aligned}
$$

(7) La fonction $g$ définie dans l'une ou l'autre de ces références est légèrement différente: c'est $\left(\int_{0}^{1} r \log r^{-1}\left|\partial\left(P_{r} f\right) / \partial r\right|^{2} d r\right)^{1 / 2}$. Du fait que $r \log r^{-1}$ et $1-r$ sont équi valents au voisinage de 1 , on montre aisément que si l'une de ces fonctions $g$ satisfait aux inégalités $L^{p}$ il en est de même de l'autre. 


$$
\begin{aligned}
& \left|\frac{\partial}{\partial r} P_{r} f\right| \leq C(1-r)^{\delta+1} \sum_{L=1}^{\infty} L A_{L}^{\delta}\left|S_{L}^{\delta+1} f-S_{L}^{\delta} f\right| r^{L-1}, \\
& \left|\frac{\partial}{\partial r} P_{r} f\right|^{2} \leq C^{2}(1-r)^{2 \delta+2}\left(\sum_{L=1}^{\infty} L A_{L}^{\delta}\left|S_{L}^{\delta+1} f-S_{L}^{\delta} f\right|^{2} r^{L-1}\right)\left(\sum_{L=1}^{\infty} L A_{L}^{\delta} r^{L-1}\right) \text {. } \\
& \text { Comme } \Sigma_{L=1}^{\infty} L A_{L}^{\delta} r^{L-1}=(\delta+1)(1-r)^{-\delta-2} \text {, } \\
& \int_{0}^{1}\left|\frac{\partial}{\partial r} P_{r} f\right|^{2}(1-r) d r \leq C^{2} \int_{0}^{1}(1-r)^{\delta+1} \sum_{L=1}^{\infty} L A_{L}^{\delta}\left|S_{L}^{\delta+1} f-S_{L}^{\delta} f\right|^{2} r^{L-1} d r \\
& \leq C^{2} \sum_{L} L^{-1}\left|S_{L}^{\delta+1} f-S_{L}^{\delta} f\right|^{2}
\end{aligned}
$$

puisque $\int_{0}^{1}(1-r)^{\delta+1} r^{L-1} d r=O\left(L^{-\delta-2}\right)=O\left(L^{-2}\left(A_{L}^{\delta}\right)^{-1}\right)$.

Plutôt que la première inégalité du Théorème (4.1), nous allons démontrer le théorème suivant, dont elle est un cas particulier:

Théorème (4.3). Soit $\nu_{k}$ une suite de constantes positives telles que $\sup _{L \geq 1} L^{-1} \Sigma_{k=1}^{L} \nu_{k}=M<\infty$ et soit

$$
g_{\delta}^{*}(f)=\left(\sum_{L=1}^{\infty}\left|S_{L}^{\delta+1} f-S_{L}^{\delta} f\right|^{2} L^{-1} \nu_{L}\right)^{1 / 2} .
$$

Sous les bypotbieses $d u$ Théorème (4.1), il existe une constante $A_{p}$ ne dépendant pas de la suite $\nu_{k}$ telle que, pour toute fonction $f \in L^{p}\left(\Sigma_{n}\right),\left\|g_{\delta}^{*}(f)\right\|_{p} \leq M A_{p}\|f\|_{p}$.

Le Théoréme (4.3) a été démontré par Muckenhoupt et Stein [17] dans le cas $\delta=0$, et sa démonstration suit un schéma bien connu (voir [25, Chapitre 15]). Le fait que $\delta \neq 0$ entraine certaines complications et nécessite la démonstration de deux lemmes combinatoires:

Lemme (4.4). Soit $\delta \geq 0, u_{k}$ une suite de nombres complexes, $s_{L}^{\delta}=$ $\left(A_{L}^{\delta}\right)^{-1} \Sigma_{l=0}^{L} A_{L-l}^{\delta} u_{l}$ et $\sigma_{L}^{\delta}=\left(A_{L}^{\delta}\right)^{-1} \Sigma_{l=0}^{L} A_{L-l}^{\delta} r^{l} u_{l}$, où $1-1 / L \leq r<1$. Alors $s_{L}^{\delta}$ peut s'écrire sous la forme

avec $a_{l, L}^{\delta}=O(1-r)$.

$$
s_{L}^{\delta}=r^{-L} \sigma_{L}^{\delta}+\sum_{l=0}^{L-1} a_{l, L}^{\delta} \sigma_{l}^{\delta}
$$

$$
\Sigma_{L=0}^{\infty} A_{L}^{\delta} s_{L}^{\delta} w^{L}=(1-w)^{-\delta-1} \Sigma_{L=0}^{\infty} u_{L} w^{L} \text { par définition des } s_{L}^{\delta} \text {. Donc }
$$




$$
\begin{aligned}
A_{L=0}^{\delta} s_{L}^{\delta} w^{L} & =(1-w)^{-\delta-1}\left(1-\frac{w}{r}\right)^{\delta+1} \sum_{L=0}^{\infty} A_{L}^{\delta} \sigma_{L}^{\delta}\left(\frac{w}{r}\right)^{L} . \\
A_{L}^{\delta} s_{L}^{\delta} & \left.=A_{l=0}^{L} A_{l}^{\delta}-l \sigma_{l}^{\delta} \sum_{k+j=L-l} A_{k}^{\delta} A_{j}^{-\delta-2} r^{-j}\right) .
\end{aligned}
$$

Si $l=L, \Sigma_{k+j=L-l} A_{k}^{\delta} A_{j}^{-\delta-2} r^{-j}=1$.

Si $l=L-1, \Sigma_{k+j=L-l} A_{k}^{\delta} A_{j}^{-\delta-2} r^{-j}=A_{1}^{\delta}+A_{1}^{-\delta-2} r^{-1}=(\delta+1)(1-1 / r)$.

Nous allons montrer que, si $2 \leq M \leq L, \sum_{j=0}^{M} A_{M-j}^{\delta_{j}} A_{j}^{-\delta-2} r^{M-j}=O(1-r)$ ce qui entrainera le lemme puisque $r^{-L} \leq(1-1 / L)^{-L} \leq$ e. Mais $\sum_{j=0}^{J} A_{j}^{a}=A_{J}^{a+1}$, et $A_{k}^{a} r^{k}-A_{k-1}^{a} r^{k-1}=r^{k} A_{k}^{\alpha-1}-(1-r) r^{k-1} A_{k-1}^{\alpha}$. Après une sommation par parties, on obtient: $\Sigma_{j=0}^{M} A_{M-j}^{\delta} A_{j}^{-\delta-2} r^{M-j}=\sum_{j=0}^{M} A_{M-j}^{\delta-1} A_{j}^{-\delta-1} r^{M-j}-(1-r) \sum_{j=0}^{M-1} A_{M-j-1}^{\delta} A_{j}^{-\delta-1} r^{M-j-1}$. Soit $k$ l'entier tel que $k-1<\delta+1 \leq k$. Après $k$ sommations par parties, on obtient des sommes de termes de la forme:

$$
(1-r)^{b} \sum_{j=0}^{M-b} A_{M-b-j}^{\delta-k+b} A_{j}^{-\delta-2+k} r^{M-b-j}, \quad b=0,1, \cdots, k
$$

dont nous allons montrer que chacune est $O(1-r)$.

Soit d'abord $b=0$ : comme $M \geq 2, \sum_{j=0}^{M} A_{M-j}^{\delta-k} A_{j}^{-\delta-2+k}=A_{M}^{-1}=0$.

Le terme étudié est donc égal à $\sum_{j=0}^{M} A_{M-j}^{\delta-k} A_{j}^{-\delta-2+k}\left(r^{M-j}-1\right)$ qui est majoré en module par $A(1-r) \sum_{j=0}^{M-1}(M-j)^{\delta-k+1} j^{-\delta-2+k}=O(1-r)$. Soit maintenant $b \geq 1: \sum_{j=0}^{M-b} A_{M-b-j}^{\delta-k+b} A_{j}^{-\delta-2+k}=A_{M-b}^{b-1}=O\left(M^{b-1}\right)$. Donc

$$
\begin{aligned}
&(1-r)^{b} \sum_{j=0}^{M-b} A_{M-b-j}^{\delta-k+b} A_{j}^{-\delta-2+k} r^{M-b-j} \\
& \leq A\left[(1-r)^{b} M^{b-1}+(1-r)^{b+1} \sum_{j=0}^{M-b}(M-b-j)^{\delta-k+b+1} j^{-\delta-2+k}\right] \\
& \quad=O(1-r) .
\end{aligned}
$$

Lemme (4.5). Soit $\delta \geq 0, u_{k}$ une suite de nombres complexes, $s_{L}^{\delta}=$ $\left(A_{L}^{\delta}\right)^{-1} \Sigma_{l=0}^{L} A_{L-l}^{\delta} u_{l}$, et $\sigma_{L}^{\delta}=\left(A_{L}^{\delta}\right)^{-1} \Sigma_{l=0}^{L} A_{L-l}^{\delta} r^{l} u_{l}$, òu $0<r<1$. Alors $\sigma_{L}^{\delta}$ peut s'écrire sous la forme:

$$
\sigma_{2}^{\delta}=\sum_{l=0}^{L} b_{l, L}^{\delta} s_{l}^{\delta} \quad \text { où } \sum_{l=0}^{L}\left|b_{l, L}^{\delta}\right|=O(1) .
$$

On montre comme dans le Lemme (4.4) que

$$
A_{L}^{\delta} \sigma_{L}^{\delta}=\sum_{l=0}^{L} A_{l}^{\delta} s_{l}{ }_{l}^{r} l \sum_{j=0}^{L-l} A_{L-l-j}^{\delta} A_{j}^{-\delta-2} r^{j}
$$


si $l=L, \sum_{j=0}^{L-l} A_{L-l-j}^{\delta} A_{j}^{-\delta-2} r^{j}=1$, si $l=L-1, \sum_{j=0}^{L-l} A_{L-l-j}^{\delta} A_{j}^{-\delta-2} r^{j}=$ $(\delta+1)(1-r)$. Soit $M \geq 2, k-1<\delta \leq k$.

Si $b=0$,

$$
\sum_{j=0}^{M} A_{M-j}^{\delta} A_{j}^{-\delta-2} r^{j}=\sum_{b=0}^{k} a_{b}(1-r)^{b} r^{b} \sum_{j=0}^{M-b} A_{M-j-b}^{\delta-k+b} A_{j}^{-\delta-2+k} r^{j} .
$$

$$
\left|\sum_{j=0}^{M} A_{M-j}^{\delta-k} A_{j}^{-\delta-2+k_{r} j}\right| \leq A(1-r) \sum_{j=0}^{M}(M-j)^{\delta-k_{j}}-\delta-1+k=O(1-r) .
$$

Si $1 \leq b \leq k-1$,

Si $b=k$,

$$
\begin{aligned}
& (1-r)^{b} r^{b}\left|\sum_{j=0}^{M-b} A_{M-j}^{\delta-k+b} A_{j}^{-\delta-2+k} r^{j}\right| \\
& \leq A(1-r)^{b+1} \sum_{j=0}^{M-b}(M-b-j)^{\delta-k+b} j^{-\delta-1+k}+(1-r)^{b}(M-b)^{b-1} .
\end{aligned}
$$

$$
\sum_{j=0}^{M-k} A_{M-k-j}^{\delta} A_{j}^{-\delta-2+k} r^{j}=(M-k)^{k-1}+\sum_{j=1}^{M-k} A_{M-k-j}^{\delta} A_{j}^{-\delta-2+k\left(r^{j}-1\right) .}
$$

Les termes de cette dernière somme étant tous positifs, elle est majorée par:

$$
(M-k)^{\delta} \sum_{j=1}^{M-k} A_{j}^{-\delta-2-k\left(r^{j}-1\right)}=(M-k)^{\delta}(1-r)^{\delta+1-k} .
$$

En conclusion:

$$
\begin{aligned}
\left|b_{l, L}^{\delta}\right| \leq A\left(\frac{l}{L}\right)^{\delta} r^{l}\left[\sum_{b=0}^{\inf (k-1, L-l)}(1-r)^{b+1}(L-l-b)^{b}\right. & \left.+(1-r)^{k}(L-l-k)^{k-1}+(1-r)^{\delta+1}(L-l-k)^{\delta}\right] \\
& \leq A\left[\sum_{b=0}^{k-1} l^{b}(1-r)^{b+1}+l^{\delta}(1-r)^{\delta+1}\right]
\end{aligned}
$$

puisque $(l / L)^{\delta}(L-l-k)^{k-1} \leq l^{k-1}$. On en déduit immédiatement que $\Sigma\left|b_{l, L}^{\delta}\right|=O(1)$.

Le lemme suivant est une conséque nce du Lemme (4.5) et est bien connu dans le cas où $\delta=0$ [25, Chapitre 15].

Lemme (4.6). Soit $r_{k}$ une suite de nombres compris entre 0 et $1, \delta_{k}$ une suite de sous-intervalles de $\left[r_{k}, 1\right.$ [. Sous les bypotbieses du Théoreme (4.1), il 
existe une constante $A_{p}$ indépendante des suites $r_{k}$ et $\delta_{k}$ telle que, pour toute suite de fonctions $f_{k}$ et toute suite d'entiers $L_{k}$,

$$
\left\|\left(\sum\left|S_{L_{k}}^{\delta} P_{r_{k}} f_{k}\right|^{2}\right)^{1 / 2}\right\|_{p} \leq A_{p}\left\|\left(\sum \frac{1}{\left|\delta_{k}\right|} \int_{\delta_{k}}\left|P_{r} f_{k}\right|^{2} d r\right)^{1 / 2}\right\|_{p} .
$$

Il suffit de montrer que, pour toutes suites $f_{k}$ et $L_{k}$,

$$
\left\|\left(\sum\left|S_{L_{k}}^{\delta} P_{r_{k}} f_{k}\right|^{2}\right)^{1 / 2}\right\|_{p} \leq A_{p}\left\|\left(\sum\left|f_{k}\right|^{2}\right)^{1 / 2}\right\|_{p}
$$

l'inégalité précédente s'en déduisant en approchant l'intégrale par des sommes de Riemann. Mais

$$
\begin{gathered}
\left|S_{L_{k}}^{\delta} P_{r_{k}} f_{k}\right|^{2} \leq A \sum_{l=0}^{L_{k}}\left|b_{l, L_{k}}^{\delta}\right|\left|S_{l}^{\delta} f_{k}\right|^{2} \\
\left\|\left(\sum\left|S_{L_{k}}^{\delta} P_{r_{k}} f_{k}\right|^{2}\right)^{1 / 2}\right\|_{p} \leq A_{p}\left\|\left(\sum_{k} \sum_{l=0}^{L_{k}}\left|b_{l, L_{k}}^{\delta}\right|\left|f_{k}\right|^{2}\right)^{1 / 2}\right\|_{p} \leq A_{p}^{\prime}\left\|\left(\sum_{k}\left|f_{k}\right|^{2}\right)^{1 / 2}\right\|_{p}
\end{gathered}
$$

Revenons à la démonstration du Théorème (4.3). On peut toujours supposer que $L \leq \Sigma_{k=1}^{L} \nu_{k} \leq 2 L$. En effet, si on a démontré le théorème dans ce cas, on l'aura démontré pour $\nu_{k} \equiv 1$, puis pour $M \leq 1$, la suite $\nu_{k}+1$ satisfaisant alors à cette condition, et donc pour toute suite $\nu_{k}$.

Posons $\mu_{1}=1, \mu_{L}=1+\sum_{l=1}^{L-1} \nu_{l}$ si $L>1, r_{L}=1-1 / \mu_{L} \cdot r_{L}$ satisfait aux hypothèses du Lemme (4.4). Or, si $f_{L}=P_{r_{L}} f$,

$$
\begin{gathered}
S_{L}^{\delta+1} f-S_{L}^{\delta} f \sim L^{-1}\left(A_{L}^{\delta}\right)^{-1} \sum_{k=0}^{L} k A_{L-k}^{\delta} H_{k} f, \\
S_{L}^{\delta+1} f_{L}-S_{L}^{\delta} f_{L} \sim L^{-1}\left(A_{L}^{\delta}\right)^{-1} \sum_{k=0}^{L} k A_{L-k}^{\delta} r_{L}^{k} H_{k} f,
\end{gathered}
$$

si bien que, en vertu du Lemme (4.4) et de l'inégalité de Schwarz,

$$
\left|S_{L}^{\delta+1} f-S_{L}^{\delta} f\right|^{2} \leq A\left(\left|S_{L}^{\delta+1} f_{L}-S_{L}^{\delta} f_{L}\right|^{2}+\frac{L}{\mu_{L}^{2}} \sum_{l=1}^{L-1} l^{2} / L^{2}\left|S_{l}^{\delta+1} f_{L}-S_{l}^{\delta} f_{L}\right|^{2}\right) .
$$

Montrons tout d'abord que

$$
\left\|\left(\sum_{L=1}^{\infty} L^{-1}\left|S_{L}^{\delta+1} f_{L}-S_{L}^{\delta} f_{L}\right|^{2} \nu_{L}\right)^{1 / 2}\right\|_{p} \leq A_{p}\|g(f)\|_{p}
$$

$S_{l}^{\delta+1} f_{L}-S_{l}^{\delta} f_{L} \sim l^{-1} S_{l}^{\delta}\left(\partial P_{r_{L}} f / \partial r\right)$, donc, en vertu du Lemme (4.6),

$$
\left\|\left(\sum_{L=1}^{\infty} L^{-1}\left|S_{L}^{\delta+1} f_{L}-S_{L}^{\delta} f_{L}\right|^{2} \nu_{L}\right)^{1 / 2}\right\|_{p} \leq A_{p}\left\|\left(\sum \frac{\nu_{L}}{L^{3}} \frac{1}{r_{L+1}-r_{L}} \int_{r_{L}}^{r}{ }^{+1}\left|\frac{\partial}{\partial r} P_{r} f\right|^{2} d r\right)^{1 / 2}\right\|_{p} .
$$


Pour obtenir l'inégalité cherchée, il suffit de remarquer que $r_{L+1}-r_{L}=\nu_{L} / \mu_{L} \mu_{L+1}$, et $1 / \mu_{L+1}=1-r_{L+1}<1-r$, si $r \in\left[r_{L}, r_{L+1}\right]$. De même:

$$
\begin{aligned}
& \left\|\left(\sum_{L} \frac{\nu_{L}}{\mu_{L}^{2}} \sum_{l=1}^{L-1} l^{2} / L^{2}\left|S_{l}^{\delta+1} f_{L}-S_{l}^{\delta} f_{L}\right|^{2}\right)^{1 / 2}\right\|_{p} \\
& \leq A_{p}\left\|\left(\sum_{L} \frac{\nu_{L}}{\mu_{L}^{2}} \sum_{l=1}^{L-1} L^{-2} \frac{1}{r_{L+1}-r_{L}} \int_{r_{L}}^{r}{ }^{r}\left|\frac{\partial}{\partial r} P_{r} f\right|^{2} d r\right)^{1 / 2}\right\|_{p} \\
& \leq A_{p}\left\|\left(\sum_{L} L^{-1} \frac{\mu_{L+1}^{2}}{\mu_{L}} \int_{r_{L}}^{r}{ }_{L+1}\left|\frac{\partial}{\partial r} P_{r} f\right|^{2}(1-r) d r\right)^{1 / 2}\right\|_{p} \leq A_{p}\|g(f)\|_{p}
\end{aligned}
$$

puisque $\mu_{L+1} / \mu_{L} \leq 2$.

Théorème (4.7). Sous les bypothèeses du Théorème (4.1), et sous l'bypotbèse supplémentaire que $\delta$ est entier, si la suite $\mu_{j}$ satisfait aux conditions:

$\left(A_{0}\right) \sup _{j}\left|\mu_{j}\right| \leq M<\infty$,

$\left(A_{k}\right) \sup _{j} 2^{j(k-1)} \Sigma_{l=2^{j}}^{2^{j+1}}\left|\Delta^{k} \mu_{l}\right| \leq M<\infty$, avec $k=\delta+1$,

alors $\mu_{j}$ définit un multiplicateur zonal de $L^{p}\left(\Sigma_{n}\right)$ : il existe une constante $A_{p}$ ne dépendant pas de $M$ telle que, pour toute fonction $f \in L^{p}\left(\Sigma_{n}\right),\left\|\sum_{j=0}^{\infty} \mu_{j} H_{j} f\right\|_{p} \leq$ $M A_{p}\|f\|_{p}$.

Posons $F=\Sigma_{j=0}^{\infty} \mu_{j} H_{j} f$. Il suffit de montrer que

$$
g_{\delta}(F) \leq A\left(\sum\left|S_{L}^{\delta+1} f-S_{L}^{\delta} f\right|^{2} \nu_{L} L^{-1}\right)^{1 / 2}
$$

où $\nu_{L}$ est une suite convenable. On peut toujours supposer $M=1$. La démonstration repose sur le lemme suivant:

Lemme (4.8). Soit $\delta$ un entier positif ou nul, $u_{k}$ une suite de nombres complexes, $s_{L}^{\delta}=\left(A_{L}^{\delta}\right)^{-1} \Sigma_{l=0}^{L} A_{L-l}^{\delta} u_{l}, \tau_{L}^{\delta}=\left(A_{L}^{\delta}\right)^{-1} \Sigma_{l=0}^{L} A_{L-l}^{\delta} \mu_{l} u_{l}$, la suite $\mu_{l}$ étant supposée satisfaire les conditions $\left(\mathrm{A}_{0}\right)$ et $\left(\mathrm{A}_{\delta+1}\right)$ avec $M=1$. Alors $\tau_{L}^{\delta}$ peut s'écrire sous la forme: $\tau_{L}^{\delta}=\mu_{L} s_{l}^{\delta}+\Sigma_{l=0}^{L-1} c_{l, L}^{\delta} s_{l}^{\delta}$, avec $c_{l, L}^{\delta}=O\left(\sum_{k=1}^{\delta+1} l^{k-1}\left|\Delta^{k} \mu_{l}\right|\right)$.

Remarquons tout d'abord que l'hypothè se sur $\mu_{j}$ entraine que

$$
\begin{aligned}
& \sum_{l=0}^{L} l^{k}\left|\Delta^{k} \mu_{l}\right|=O(L), \quad k=0,1,2, \cdots, \delta+1 . \\
& \sum_{L} A_{L}^{\delta} \tau_{L}^{\delta} w^{L}=(1-w)^{-\delta-1} \sum_{l} \mu_{l} w^{l} u_{l}=(1-w)^{-\delta-1} \sum_{l} \Delta^{\delta+1}\left(\mu_{l} w^{l}\right) A_{l}^{\delta} s_{l}^{\delta} . \\
& \Delta^{\delta+1}\left(\mu_{l} w^{l}\right)=\sum_{k=0}^{\delta+1} A_{k}^{-\delta-2} \Delta^{k} \mu_{l} \Delta^{\delta+1-k} w^{l+k}=\sum_{k=0}^{\delta+1} A_{k}^{-\delta-2} \Delta^{k} \mu_{l} w^{l+k}(1-w)^{\delta+1-k} .
\end{aligned}
$$




$$
A_{L}^{\delta} \tau_{L}^{\delta}=\sum_{l=0}^{L} A_{l}^{\delta} s_{l}^{\delta} \sum_{k+j=L-l ; k \leq \delta+1} \Delta^{k} \mu_{l} A_{k}^{-2-\delta} A_{j}^{k-1}
$$

$\Sigma_{k+j=L-l} A_{k}^{-\delta-2} A_{j}^{k-1} \Delta^{k} \mu_{l}=\mu_{l}$ si $l=L$. Si $l \leq L-1$,

$$
\left|\sum_{k+j=L-l ; k \leq \varepsilon+1} A_{k}^{-\delta-2} A_{j}^{k-1} \Delta^{k} \mu_{l}\right| \leq A \sum_{k=1}^{\delta+1}(L-l)^{k-1}\left|\Delta^{k} \mu_{l}\right| .
$$

Donc $\left|c_{l, L}^{\delta}\right| \leq A l^{\delta} L^{-\delta} \sum_{k=1}^{\delta+1}(L-l)^{k-1}\left|\Delta^{k} \mu_{l}\right| \leq A \Sigma_{k=1}^{\delta+1} l^{k-1}\left|\Delta^{k} \mu_{l}\right|$.

Revenons à la démonstration du théorème.

$$
\begin{aligned}
\left|g_{\delta}(F)\right|^{2} & \leq \sum_{L}\left|S_{L}^{\delta+1} f-S_{L}^{\delta} f\right|^{2} L^{-1}+A \sum_{L} L^{-2} \sum_{l=0}^{L-1}\left|S_{l}^{\delta+1} f-S_{l}^{\delta} f\right|^{2} l\left|c_{l, L}^{\delta}\right| . \\
& \leq \sum_{L}\left|S_{L}^{\delta+1} f-S_{L}^{\delta} f\right|^{2} L^{-1}+A\left(\sum_{l}\left|S_{l}^{\delta+1} f-S_{l}^{\delta} f\right|^{2} \frac{\nu_{l}}{l}\right)
\end{aligned}
$$

avec $\nu_{l}=\Sigma_{k=1}^{\delta+1} l^{k}\left|\Delta^{k} \mu_{l}\right|$, du fait que $\sum_{l=0}^{L-1} l\left|c_{l, L}^{\delta}\right|=O(L)$, et de la majoration sur $c_{l, L}^{\delta}$.

Théorème (4.9). (1) Soit $\mu_{j}$ une suite satisfaisant aux conditions:

$\left(A_{0}\right) \sup _{j}\left|\mu_{j}\right| \leq M<\infty$,

$\left(A_{k}\right) \sup _{j} 2^{j(k-1)} \Sigma_{l=2^{j}}^{2^{j+1}}\left|\Delta^{k} \mu_{l}\right| \leq M<\infty$,

avec $k=(n+1) / 2$ si $n$ est impair, $k=(n+2) / 2$ si $n$ est pair. La suite $\mu_{i}$

définit un multiplicateur de $L^{p}\left(\Sigma_{n}\right)$ pour tout $p>1$, dont la norme est bornée par $M A_{p}$, où $A_{p}$ ne dépend pas de $\mu_{j}$.

(2) Soit $\mu_{j}$ une suite satisfaisant aux conditions $\left(A_{0}\right)$ et $\left(A_{k}\right)$, où $2 \leq k<$ $(n+1) / 2$. Elle définit un multiplicateur de $L^{p}\left(\Sigma_{n}\right)$ pour $|1 / p-1 / 2|<(k-1) /(n-1)$.

C'est une conséquence immédiate du Théorème (4.7), du Théorème (3.6) et du Corollaire (3.7). En (1) est exprimée la condition suffisante de ce type la plus faible pour que $\mu_{j}$ soit un multiplicateur, comme le montre la considération des moyennes de Cesàro (autrement dit la condition $\left(A_{k}\right)$ ne peut être remplacée par $\left(A_{k-1}\right)$ ). Ce n'est sûrement pas le cas pour (2) (voir $\$ 5$ ).

Remarque. Le Theorème (4.1) permet également d'énoncer des conditions suffisantes de type Hörmander pour que $\mu_{j}$ soit un multiplicateur, et de géneraliser ainsi les résultats de [3] à $\Sigma_{n}$ : en effet, en interpolant suivant une idée d'Igari et Kuratsubo [14] entre les deux résultats:

on obtient que

$$
\begin{aligned}
&\left\|g_{(n-1) / 2}(f)\right\|_{p} \leq A_{p}\|f\|_{p} \text { si } p>1 \\
&\left\|g_{\delta}(f)\right\|_{2} \leq A_{2}\|f\|_{2} \quad \text { si } \delta>-1 / 2
\end{aligned}
$$




$$
\left\|g_{\delta}(f)\right\|_{p} \leq A_{p}\|f\|_{p} \quad \text { si }|1 / p-1 / 2|<(2 \delta+1) / 2 n \text {. }
$$

En utilisant le Lemme (4.8) on en déduit le théorème suivant:

Théorème (4.10). Soit $\mu_{j}$ une suite satisfaisant aux conditions suivantes:

$\left(\mathrm{B}_{0}\right) \sup \left|\mu_{j}\right|<\infty$,

$\left(B_{k}\right) \sup 2^{j(2 k-1)} \Sigma_{2^{j}}^{2^{j+1}}\left|\Delta^{k} \mu_{j}\right|^{2}<\infty$.

Alors $\mu_{j}$ définit un multiplicateur de $L^{p}\left(\Sigma_{n}\right)$ pour les valeurs de $p$ supérieures d 1 telles que $|1 / p-1 / 2|<(2 k-1) / 2 n(k \geq 1)$.

Sauf lorsque $k>(n+1) / 2$, les conditions des Théorèmes (4.9) et (4.10) ne sont pas comparables. Remarquons toutefois que $\left(A_{0}\right)$ et $\left(A_{k}\right)$ entrainent $\left(B_{k-1}\right)$.

Le théorème de passage de $\Sigma_{n}$ à $\mathbf{R}^{n}$ permet de déduire immédiatement des théorèmes (4.9) et (4.10) leurs analogues sur $\mathbf{R}^{n}$.

Théorème (4.11). Soit $k$ un entier supérieur ou égal à $1, m$ une fonction bornée sur $\mathbf{R}^{+}$de classe $\mathcal{C}^{k}$, qui satisfait à la condition:

$$
\sup _{j} \int_{2^{j}}^{2^{j+1}}\left|m^{(k)}(x)\right| d x \leq A 2^{-j(k-1)} \text {. }
$$

La fonction $m$ définit un multiplicateur radial de $L^{p}\left(\mathbf{R}^{n}\right)$ pour $|1 / p-1 / 2|<$ $(k-1) /(n-1), p>1$.

Théorème (4.12). Soit $k$ un entier supérieur ou égal à $1, m$ une fonction bornée sur $\mathbf{R}^{+}$de classe $\mathcal{C}^{k}$, qui satisfait à la condition:

$$
\sup _{j} \int_{2^{j}}^{2^{j+1}}\left|m^{(k)}(x)\right|^{2} d x \leq A 2^{-j(2 k-1)} .
$$

La fonction $m$ définit un multiplicateur radial de $L^{p}\left(\mathbf{R}^{n}\right)$ pour $|1 / p-1 / 2|<$ $(2 k-1) / 2 n$.

En effet, les suites $m_{l}^{\epsilon}$, définies par $m_{l}^{\epsilon}=m(\epsilon l)$, satisfont uniformément aux hypothèses des Théorèmes (4.9) et (4.10) respectivement: il suffit de remarquer que $\left|\Delta^{k} m_{l}^{\epsilon}\right| \leq \epsilon^{k-1} \int_{\epsilon l}^{\epsilon(l+k)}\left|m^{(k)}(x)\right| d x$. Le Théorème (4.12), dans le cas où $k=1$, est dû à Igari et Kuratsubo [14].

5. Etude des sommes de Cesàro en dessous de l'indice critique $(n \geq 2)$. Il est bien connu que si $\delta>(n-1) / 2$, alors les sommes de Cesàro d'indice $\delta$ sont uniformément bornées dans $L^{p}\left(\Sigma_{n}\right)(1 \leq p \leq+\infty)$, et par suite $S_{L}^{\delta} f \rightarrow f$ dans $L^{p}$ $(1 \leq p<+\infty)$. Si maintenant $\delta \leq(n-1) / 2$, on ne peut plus espérer un résultat positif pour tout indice $p$. En effet:

Théorème (5.1). (i) Soit $\delta=0$; il existe, pour tout $p \neq 2$, une fonction $f \in L^{p}\left(\Sigma_{n}\right)$, telle que $S_{L}^{0} f$ ne converge pas dans $L^{p}\left(\Sigma_{n}\right)$.

(ii) Soit $0^{\circ}<\delta \leq(n-1) / 2$; il existe, pour tout $p, 1 \leq p \leq 2 n /(n+1+2 \delta)$ ou 
$p \geq 2 n /(n-1-2 \delta)$, une fonction $f \in L^{p}\left(\Sigma_{n}\right)$, telle que $S_{L}^{\delta} f$ ne converge pas dans $L^{p}\left(\Sigma_{n}\right)$.

En effet, la convergence dans $L^{p}$ de $\left(S_{L}^{\delta} f\right)_{L \geq 0}$ pour toute $f \in L^{p}$ est équivalente au fait que les opérateurs $\left(S_{L}^{\delta}\right)_{L \geq 0}$ sont uniformément bornés dans $L^{p}$. Le théorème résulte alors du Théorème (1.1) et des résultats correspondants pour $\mathrm{R}^{n}$ (voir [7], [21]). Remarquons d'ailleurs que seul le résultat (i) est réellement nouveau; en effet, pour (ii) on peut trouver une fonction zonale $f$, telle que $S_{L}^{\delta} f$ ne converge pas dans $L^{p}\left(\Sigma_{n}\right)[1]$.

Ces résultats amènent à la conjecture naturelle que dans l'intervalle complémentaire en $p$, les sommes de Cesàro sont uniformément bornées. Nous montrons ici l'analogue du résultat de Fefferman pour $\mathbf{R}^{n}$ (voir [6]), id est la réponse à la conjecture précédente est positive si $\delta>(n-1) / 4$.

Théorème (5.2). Soit $\delta,(n-1) / 4<\delta \leq(n-1) / 2$; pour tout $p, 2 n /(n+1+2 \delta)$ $<p<2 n /(n-1-2 \delta)$, il existe une constante $A_{p}$ telle que $\left\|S_{L}^{\delta} f\right\|_{p} \leq A_{p}\|f\|_{p}$.

La démonstration repose sur un lemme, analogue du lemme de restriction de [6].

Lemme (5.3). Soit $p, 1 \leq p<4 n /(3 n+1)$; alors $\left\|H_{k} f\right\|_{2} \leq C \cdot k^{n(1 / p-(n+1) / 2 n)}\|f\|_{p}$.

Remarque. On peut, a priori, penser à interpoler entre les deux inégalités

$$
\left\|H_{k} f\right\|_{2} \leq C\|f\|_{2} \text { et }\left\|H_{k} f\right\|_{2} \leq C \cdot\left\|Z_{k}\right\|_{2} \cdot\|f\|_{1},
$$

notant que $\left\|Z_{k}\right\|_{2}=O\left(k^{(n-1) / 2}\right)$. Mais l'inégalité obtenue est plus mauvaise que celle du lemme.

Démonstration. $\left\|H_{k} f\right\|_{2}^{2}=\int_{\boldsymbol{\Sigma}_{n}}\left(H_{k} f\right)(\xi) \overline{f(\xi)} d \sigma(\xi) \leq\|f\|_{p} \cdot\left\|Z_{k} * f\right\|_{p^{\prime}} \leq$ $\|f\|_{p} \cdot\|f\|_{p}\left\|Z_{k}\right\|_{q}$, où $1 / p^{\prime}=1 / p+1 / q-1$ d'après les inégalités de Hölder et de Young. Notons que $q=p^{\prime} / 2>2 n /(n-1)=2+1 / \lambda$. Estimons maintenant $\left\|Z_{k}\right\|_{q}$; rappelons que ${ }^{b} \mathrm{Z}_{k}=((k+\lambda) / \lambda) P_{k}^{\lambda}$, et que $P_{k}^{\lambda}$ satisfait aux estimations suivantes (voir [22, p. 170]).

De sorte que

$$
\left|P_{k}^{\lambda}(\cos \theta)\right| \leq \begin{cases}C \cdot \theta^{-\lambda} k^{\lambda-1} & \text { si } 1 / k \leq \theta \leq \pi / 2 \\ k^{2 \lambda-1} & \text { si } 0 \leq \theta \leq 1 / k\end{cases}
$$

$$
\begin{aligned}
& \int_{\Sigma_{n}}\left|Z_{k}(\xi)\right|^{q} d \sigma(\xi)=O\left(k^{q}\right) \int_{0}^{\pi}\left|P_{k}^{\lambda}(\cos \theta)\right|^{q}(\sin \theta)^{2 \lambda} d \theta \\
& =O\left(k^{q}\right)\left(\int_{0}^{1 / k} k^{(2 \lambda-1) q} \theta^{2 \lambda} d \theta+\int_{1 / k}^{\pi / 2} k^{(\lambda-1) q} \theta^{-\lambda q} \theta^{2 \lambda} d \theta\right) \\
& =O\left(k^{2 \lambda q-2 \lambda-1}\right), \text { puisque } \lambda q>2 \lambda+1 .
\end{aligned}
$$

D'où $\left\|Z_{k}\right\|_{q} \leq C \cdot k^{2 n(1 / p-(n+1) / 2 n)}$.

Cela étant, soit donc $\delta,(n-1) / 4<\delta \leq(n-1) / 2$, et posons $p_{0}=4 n /(3 n+1)$, 
$p_{\delta}=2 n /(n+1+2 \delta)$, de sorte que $p_{\delta}<p_{0}$; soit donc $p, p_{\delta}<p<p_{0}$; nous devons montrer que $\left\|S_{L}^{\delta} f\right\|_{p} \leq A_{p}\|f\|_{p}$.

On va d'abord retrancher à $S_{L}^{\delta}$ une partie régulière; pour ceci soit $\theta$ une fonction de classe $C^{\infty}$ sur $R$, satisfaisant

(i) $0 \leq \theta \leq 1$,

(ii) $\theta=0$ si $t \leq 2 / 3$ ou si $t \geq 2$,

(iii) $\theta=1$ si $3 / 4 \leq t \leq 1$.

Si $\phi$ est une fonction zonale sur $\Sigma_{n}$, nous noterons dans ce paragraphe $\hat{\phi}(k)$ ses "coefficients de Fourier", id est $\phi=\Sigma_{0}^{+\infty} \hat{\phi}(k) Z_{k}$. En particulier $s_{L}^{\delta}=$ $\Sigma_{0}^{L} \hat{s}_{L}^{\delta}(k) Z_{k}$, avec $\hat{s}_{L}^{\delta}(k)=A_{L-k}^{\delta} / A_{L}^{\delta}$. On décompose alors $s_{L}^{\delta}$ à l'aide de $\theta$ en posant $s_{L}^{\delta^{k}}=s_{L}^{\prime}+s_{L}^{\prime \prime}$, où $\hat{s}_{L}^{\prime}(k)=\theta(k / L) \hat{s}_{L}^{\delta}(k)$; de sorte que $S_{L}^{\delta}=S_{L}^{\prime}+S_{L}^{\prime \prime}$, où $S_{L}^{\prime}$ contient la singularité véritable de $S_{L}^{\delta}$ et $S_{L}^{\prime \prime}$ n'est qu'un terme trivial; montrons en effet que $\left\|S_{L}^{\prime \prime} f\right\|_{p} \leq C \cdot\|f\|_{p}$. Pour ceci on évalue les différences successives de $\hat{s}_{L}^{\prime \prime}(k)$, et on va voir que le théoréme de multiplicateurs (4.9) fournit le résultat avec une constante uniforme; en effet

(i) $\left|\Delta^{r} \theta(k / L)\right| \leq C \cdot L^{-r}$,

(ii) $\left|\Delta^{s} A_{L-k}^{\delta}\right| / A_{L}^{\delta} \leq C \cdot L^{-s}$, dès lors que $L-k \geq L / 4$.

(i) résulte de la formule des accroissements finis, et (ii) de la formule

$$
\begin{aligned}
\Delta^{s} A_{L-k}^{\delta} & =\Gamma(L-k+\delta+1-q) / \Gamma(L-k+1) \Gamma(\delta-q+1) \\
& =0 \text { sinon, }
\end{aligned}
$$

qui se démontre par récurrence.

La formule de Leibniz montre a lors que $\left|\Delta^{m} s_{L}^{\prime \prime}(k)\right| \leq C / k^{m}$.

Venons-en à l'estimation de $\left\|S_{L}^{\prime} f\right\|_{p}$. Fixons $L$, et soit $J$ le premier entier positif tel que $2^{J} / L \geq \pi$. Choisissons maintenant des fonctions $\left(\phi_{j}^{L}\right)_{0 \leq j \leq J}$, telles que

(i) $\phi_{j}^{L}$ est zonale, $0 \leq \phi_{j} \leq 1$,

(ii) $\Sigma \phi_{j}^{L}=1$,

(iii) le support de $\phi_{0}^{L}$ est contenu dans la boule $B(1,2 / L)$, le support de $\phi_{j}^{L}$ est contenu dans la "calotte" $\left\{\xi \mid 2^{j-1} / L<d(1, \xi) \leq 2^{j+1} / L\right\}$.

(iv) $\phi_{j}^{L}$ est de classe $C^{\infty}$, et pour tout opérateur différentiel $D$ de degré $M,\left|D \phi_{j}^{L}\right| \leq C\left(2^{j} / L\right)^{-M}$, où la constante $C$ ne dépend ni de $j$, ni de $L$.

Cette partition de l'unité permet de décomposer $s_{L}^{\prime}$ en $\Sigma_{j} s_{L}^{j}$, où $s_{L}^{j}=$ $\phi_{j} \cdot s_{L}^{\prime}$. On va montrer que $\left\|s_{L}^{j} * f\right\|_{p} \leq C \cdot 2^{-\epsilon}\|f\|_{p}$, avec un nombre $\epsilon>0$, dont la valeur sera précisée en cours de démonstration. Fixons maintenant $j(0 \leq j \leq J)$, et construisons par récurrence un recouvrement de $\Sigma_{n}$ par des boules $B\left(\xi_{i}, 2^{j} / L\right)$, telles que $d\left(\xi_{i}, \xi_{i^{\prime}}\right) \geq 2^{j} / L$ dès que $i \neq i^{\prime}$. Remarquons tout de suite que ces boules (ainsi que les boules de rayon, disons, triple) sont $N$-disjointes (en ce sens que l'intersection de plus de $N$ boules distinctes est vide), 
où $N$ ne dépend que de la dimension $n$ (ce résultat tient à la nature homogène de l'espace $\Sigma_{n}$, au sens de [5])

Soit maintenant $\left(\psi_{i}\right)$ une partition de l'unité subordonnée à ce recouvrement, et $f=\Sigma \psi_{i} f=\Sigma f_{i}$ la décomposition correspondante de $f$. Alors

$$
\left\|s_{L}^{j} f\right\|_{p}^{p}=\int\left|\sum_{i} s_{L}^{j} * f_{i}\right|^{p}(\xi) d \xi \leq N^{p-1} \sum_{i} \int\left|s_{L}^{j} * f_{i}\right|^{p}(\xi) d \xi,
$$

puisque $s_{L}^{j} * f_{i}$ a son support dans la boule $B\left(\xi_{i}, 3 \cdot 2^{j} / L\right)$. On voit alors facilement qu'il suffit de démontrer que $\left\|s_{L}^{j} * f\right\|_{p} \leq C \cdot 2^{-\epsilon j}\|f\|_{p}$ lorsque $f$ a son support contenu dans une boule de rayon $2^{j} / L$. On peut toujours supposer que cette boule est centrée au pôle nord, puisque $S_{L}^{j}$ commute aux rotations. Mais a lors $s_{L}^{j} * f$ a son support dans la boule $B\left(3 \cdot 2^{j} / L\right)$, et par suite $\left\|S_{L}^{j} f\right\|_{p} \leq$ $C \cdot\left(2^{j} / L\right)^{n(1 / p-1 / 2)}\left\|S_{L}^{j} f\right\|_{2}$. Par suite, tout revient à montrer que $\left\|S_{L}^{j} f\right\|_{2} \leq$ $C \cdot\left(2^{j} / L\right)^{-n(1 / p-1 / 2)} 2^{-j \epsilon}\|f\|_{p}$. Or $\left\|S_{L}^{j} f\right\|_{2}^{2}=\Sigma_{0}^{+\infty}\left|\hat{s}_{L}^{j}(k)\right|^{2}\left\|H_{k} f\right\|_{2}^{2} \leq$ $\left(\Sigma_{0}^{+\infty}\left|\hat{s}_{L}^{j}(k)\right|^{2} k^{2 \gamma}\right)\|f\|_{p}^{2}$, grace au Lemme (5.3), avec $\gamma=n(1 / p-(n+1) / 2 n)$; notons que $\gamma<(n-1) / 2$. Majorons d'abord $\Sigma_{0}^{L / 3}\left|\hat{s}_{L}^{j}(k)\right|^{2} k^{2 \gamma}$; c'est en fait encore un terme trivial. Rappelons que $s_{L}^{j}=\phi_{j} \cdot s_{L}^{\prime}$, de sorte qu'avec des notations évidentes, $s_{L}^{j}=\left(\Sigma \hat{s}_{L}^{\prime}(m) Z_{m}\right) \cdot\left(\Sigma \hat{\phi}_{j}(l) Z_{l}\right)$; mais $Z_{m} Z_{l}=\Sigma_{|l-m|}^{l+m} a_{m, l}^{k} Z_{k}$, où

$$
a_{m, l}^{k}=C_{\lambda} \cdot \frac{\Gamma(k+1) \Gamma(g+2 \lambda) \Gamma(g-l+\lambda) \Gamma(g-m+\lambda) \Gamma(g-k+\lambda)}{(m+\lambda)(l+\lambda) \Gamma(k+2 \lambda) \Gamma(g+\lambda+1) \Gamma(g-l+1) \Gamma(g-m+1) \Gamma(g-k+1)},
$$

avec $2 g=l+m+k$, la sommation étant faite pour les indices $k$ ayant même parité que $l+m$ (voir [23, p. 504], où la formule pour $\alpha_{m_{3} l}^{k}$ est donnée avec une légère erreur). On déduit que $\hat{s}_{L}^{j}(k)=\Sigma_{|l-m|<k<l+m} a_{m_{m}}^{k} \hat{s}_{L}^{\prime}(m) \hat{\phi}_{j}(l)$. On peut toujours supposer $m \geq 2 L / 3$, grâce au découpage de $S_{L}$ en $S_{L}^{\prime}+S_{L}^{\prime \prime}$ effectué au début, et l'on s'intéresse aux valeurs de $k \leq L / 3$. A partir de ces conditions, on estime les quantités $g, g-l, \ldots$, et on montre que $a_{m, l}^{k} \leq C_{\lambda} 1 / k L^{2 \lambda}$. Par suite $\left|\hat{s}_{L}^{j}(k)\right| \leq C \cdot L^{2 \lambda} \Sigma_{L / 3}^{4 L / 3}\left|\hat{\phi}_{j}(l)\right|$. Il reste à estimer la norme $l^{1}$ de la transformée de Fourier de $\phi_{j}$, qui est une fonction très régulière; on utilise le Laplacien $\Delta$ sur $\Sigma_{n}$, dont on sait que $Z_{l}$ est une fonction propre avec une valeur propre en $O\left(l^{2}\right)$. Par suite

$$
\begin{aligned}
\sum_{L / 3}^{4 L / 3}\left|\hat{\phi}_{j}(l)\right| & \leq C\left(\sum_{0}^{+\infty}\left|\hat{\phi}_{j}(l)\right|^{2} l^{4 M} l^{n-1}\right)^{1 / 2}\left(\sum_{L / 3}^{+\infty} l^{-4 M} l^{-(n-1)}\right)^{1 / 2} \\
& \leq C \cdot\left(\int_{\Sigma_{n}}\left|\Delta^{M} \phi_{j}\right|^{2}(\xi) d \sigma(\xi)\right)^{1 / 2} L^{-2 M-n / 2+1}
\end{aligned}
$$

où $M$ est un entier suffisamment grand, qu'on choisira dans un instant. Mais, par hypothèse $\left|\Delta^{M} \phi_{j}\right| \leq C\left(2^{j} / L\right)^{-2 M}$, d'après la propriété (iv).

$$
\left(\int_{\Sigma_{n}}\left|\Delta^{M} \phi_{j}\right|^{2}(\xi) d \sigma(\xi)\right)^{1 / 2} \leq C \cdot\left(\frac{2^{j}}{L}\right)^{-2 M} \cdot\left(\frac{2^{j}}{L}\right)^{n / 2} \text {. }
$$


D'où finalement $\left|\hat{s}_{L}^{j}(k)\right| \leq C L^{2}\left(2^{j} / L\right)^{-2 M}\left(2^{j} / L\right)^{n / 2} L-2 M-n / 2+1 \leq C \cdot 2^{j(n / 2-M)}$, et finalement $\Sigma_{0}^{L / 3}\left|\hat{s}_{L}^{j}(k)\right|^{2} k^{2 \gamma} \leq C \cdot L^{2 \gamma+1} \cdot 2^{j(n / 2-2 M)}$; or $2 \gamma+1=2 n(1 / p-1 / 2)$; il ne reste plus qu'à choisir $M$ tel que $n / 2-2 M \leq-2 n(1 / p-1 / 2)-\epsilon$. Abordons maintenant la partie réellement significative du problème

$$
\begin{aligned}
\sum_{L / 3}^{+\infty}\left|\hat{s}_{L}^{j}(k)\right|^{2} l^{2 \gamma} & \leq C \cdot L^{2 \gamma-(n-1)} \sum_{0}^{+\infty}\left|\hat{s}_{L}^{j}(k)\right|^{2}\left\|Z_{k}\right\|_{2}^{2} \\
& \leq C \cdot L^{2 \gamma-(n-1)} \int_{\Sigma_{n}}\left|s_{L}^{j}(\xi)\right|^{2} d \sigma(\xi) .
\end{aligned}
$$

On utilise les majorations du $\$ 2$, de sorte que

$$
\begin{aligned}
\int_{\Sigma_{n}}\left|s_{L}^{j}(\xi)\right|^{2} d \sigma(\xi) & \leq \int_{2^{(j-1) / L}}^{2^{(j+1)} / L} L^{2 \lambda-2 \delta}|\theta|^{-2 \lambda-2 \delta-2}|\theta|^{2 \lambda} d \theta \\
& \leq C \cdot L^{2 \lambda-2 \delta}\left(\frac{2^{j}}{L}\right)^{-2 \delta-1}=C \cdot L^{2 \lambda+1}\left(2^{j}\right)^{-2 \delta-1}
\end{aligned}
$$

pour $j=0, J-1, J$, on doit utiliser les autres inégalités $S_{L}^{\delta}$, mais la majoration obtenue est identique. En définitive, $\Sigma_{L / 3}^{+\infty}\left|\hat{s}_{L}^{j}(l)\right|^{2} l^{2 \gamma} \leq C \cdot L^{2 \gamma+1}\left(2^{j}\right)^{-2 \delta-1}$. Notant alors que $p>p_{\delta}$ entraine $\gamma<\delta$, on pose $\delta=\gamma+\epsilon$, et la majoration devient $\Sigma_{L / 3}^{+\infty}\left|\hat{s}_{L}^{j}(l)\right|^{2} l^{2 \gamma} \leq C \cdot\left(2^{j} / L\right)^{-2(1 / p-1 / 2)} \cdot 2^{-2 j \epsilon}$. D'où la majoration cherchée.

6. Polynômes ultrasphériques. Considérons l'intervalle $[0, \pi]$ muni de la distance induite de celle de $\mathbf{R}$, et, sur $[0, \pi]$, la mesure $d m_{\beta}(\theta)=(\sin \theta)^{\beta} d \theta$, où $\beta$ est un nombre réel supérieur à -1 . Il est a isé de montrer que $m_{\beta}$ satisfait à la propriété d'homogénéité: $m_{\beta}(S(\theta, r)) \leq A m_{\beta}(S(\theta, r / 2)), S(\theta, r)$ désignant ici l'ensemble $\{\phi \in[0, \pi] ;|\theta-\phi|<r\}$.

La fonction maximale $M_{\beta} f$ de $f$ relativement à la mesure $m_{\beta}$ est définie par:

$$
M_{\beta} f(\theta)=\sup _{r>0} \frac{1}{m_{\beta}(S(\theta, r))} \int_{S(\theta, r)}|f(\phi)| d m_{\beta}(\phi) .
$$

En vertu du théorème de Fefferman-Stein [8], quels que soient $r>1$ et $\mu>1$, il existe une constante $A_{r \mu}$ telle que:

$$
\left\|\left(\sum_{n}\left|M_{\beta} f_{n}\right|^{r}\right)^{1 / r}\right\|_{L}^{\mu}{ }_{\left(d m_{\beta}\right)} \leq A_{r \mu}\left\|\left(\sum\left|f_{n}\right|^{r}\right)^{1 / r}\right\|_{L_{\left(d m_{\beta}\right)}^{\mu}}
$$

pour toute suite de fonctions $f_{n}$.

Soit maintenant $\lambda$ un nombre positif fixé une fois pour toutes. Dans la suite $M$ désignera $M_{2 \lambda},\|f\|_{p}$ la norme de $f$ dans $L^{p}\left(d m_{2 \lambda}\right)$. Soit $1<p \leq 2$, $p^{\prime}$ l'exposant conjugué, $\alpha \in]_{-}(2 \lambda+1) / p,(2 \lambda+1) / p^{\prime}\left[\right.$. On notera encore $\|f\|_{p, a}$ la norme de $f(\theta)(\sin \theta)^{a}$ dans $L^{p}\left(d m_{2 \lambda}\right)$.

Soit alors $q<p$ tel que $\alpha \in]-(2 \lambda+1) / q,(2 \lambda+1) / q^{\prime}[$. Il existe une 
constante $A$ telle que, pour toute fonction $f \in L^{p}\left(d m_{a p+2 \lambda}\right), M f \leq A\left(M_{a q+2 \lambda} f^{q}\right)^{1 / q}$; en effet

$$
\begin{aligned}
& \frac{1}{m_{2 \lambda}(S(\theta, r))} \int_{S(\theta, r)}|f(\phi)| \sin ^{2 \lambda} \phi d \phi \\
& \quad \leq \frac{1}{m_{2 \lambda}(S(\theta, r))}\left(\int_{S(\theta, r)}|f(\phi)|^{q} \sin ^{\alpha q+2 \lambda} \phi d \phi\right)^{1 / q}\left(\int_{S(\theta, r)}(\sin \phi)^{-\alpha q^{\prime}+2 \lambda} d \phi\right)^{1 / q^{\prime}} \\
& \quad \leq \frac{A}{\left(m_{2 \lambda+a q}(S(\theta, r))\right)^{1 / q}}\left(\int_{S(\theta, r)}|f(\phi)|^{q} \sin ^{\alpha q+2 \lambda} \phi d \phi\right)^{1 / q} \leq A\left(M_{a q+2 \lambda} f^{q}\right)^{1 / q}
\end{aligned}
$$

en vertu de l'inégalité élémentaire:

$\left(\int_{S(\theta, r)}(\sin \phi)^{a q+2 \lambda} d \phi\right)^{1 / q}\left(\int_{S(\theta, r)}(\sin \phi)^{-a q^{\prime}+2 \lambda} d \phi\right)^{1 / q^{\prime}} \leq A \int_{S(\theta, r)} \sin ^{2 \lambda} \phi d \phi$.

Appliquons alors le théorème de Fefferman-Stein avec $r=2 / q, \mu=p / q$ : on obtient:

Lemme (6.1). Soit $1<p<\infty, a \in]-(2 \lambda+1) / p,(2 \lambda+1) / p^{\prime}[$. Il existe une constante $A_{p, a}$ telle que, pour toute suite de fonctions $f_{n},\left\|\left(\Sigma\left|M f_{n}\right|^{2}\right)^{1 / 2}\right\|_{p, a} \leq$ $A_{p, a}\left\|\left(\Sigma\left|f_{n}\right|^{2}\right)^{1 / 2}\right\|_{p, a}$.

Soit $f$ une fonction de $L^{2}\left(d m_{2 \lambda}\right)$. Les fonctions $P_{n}^{\lambda}(\cos \theta)$ formant un système orthogonal complet sur $[0, \pi]$ muni de la mesure $d m_{2 \lambda}, f$ possède un développement: $f(\theta) \sim \sum a_{n} P_{n}^{\lambda}(\cos \theta)$. Les moyennes de Cesàro d'ordre $\delta$ de $f$ sont définies par

$$
S_{L}^{\delta} f(\theta)=\left(A_{L}^{\delta}\right)^{-1} \sum_{0}^{L} A_{L-l}^{\delta} a_{l} P_{l}^{\lambda}(\cos \theta),
$$

ou encore par

$$
s_{L}^{\delta} f(\theta)=\int_{0}^{\pi} s_{L}^{\delta}(\cos \theta, \cos \phi) f(\phi) d m_{2 \lambda}(\phi)
$$

où $s_{L}^{\delta}(\cos \theta, \cos \phi)=\left(A_{L}^{\delta}\right)^{-1} \sum_{l=0}^{L} A_{L-l}^{\delta}\left\|P_{l}^{\lambda}\right\|_{2}^{-2} P_{l}^{\lambda}(\cos \theta) P_{l}^{\lambda}(\cos \phi)$. En vertu de la formule d'addition

$$
P_{n}^{\lambda}(\cos \theta) P_{n}^{\lambda}(\cos \phi)=c_{\lambda} \int_{0}^{\pi} P_{n}^{\lambda}(\cos \Omega) P_{n}^{\lambda}(1) \sin ^{2 \lambda-1} w d w
$$

où $c_{\lambda}^{-1}=\int_{0}^{\pi} \sin ^{2 \lambda-1} w d w$ et $\cos \Omega=\cos \theta \cos \phi+\cos w \sin \theta \sin \phi$,

$$
s_{L}^{\delta}(\cos \theta, \cos \phi)=c_{\lambda} \int_{0}^{\pi} s_{L}^{\delta}(\cos \Omega) \sin ^{2 \lambda-1} w d w,
$$

où $s_{L}^{\delta}(\cos \theta)=\left(A_{L}^{\delta}\right)^{-1} \Sigma_{l=0}^{L} A_{L-l}^{\delta}\left\|P_{l}^{\lambda}\right\|_{2}^{-2} P_{l}^{\lambda}(1) P_{l}^{\lambda}(\cos \theta)$ est le noyau de Cesàro étudié dans les paragraphes 2 et 3 . Rappelons les majorations obtenues: $s i$ $\sigma_{L}^{\delta}(\cos \theta)=s_{L}^{\delta}(\cos \theta) \chi[0, \pi / 2](\theta), \tau_{L}^{\delta}(\cos \theta)=s_{L}^{\delta}(\cos \theta)-\sigma_{L}^{\delta}(\cos \theta)$, 


$$
\begin{aligned}
& \left|\sigma_{L}^{\delta}(\cos \theta)\right| \leq A L^{\lambda-\delta}\left(L^{-2}+|\theta|^{2}\right)^{-(\lambda-\delta-1) / 2} \quad \text { si } \delta \leq \lambda+1 \\
& \left|\sigma_{L}^{\delta}(\cos \theta)\right| \leq A L^{-1}\left(L^{-2}+|\theta|^{2}\right)^{-\lambda-1} \quad \text { si } \delta \geq \lambda+1 \\
& \left|\tau_{L}^{\delta}(\cos \theta)\right| \leq A|\pi-\theta|^{-2 \lambda+\delta} \quad \text { si } \delta \leq 2 \lambda ; \\
& \left|\tau_{L}^{\delta}(\cos \theta)\right| \leq A \text { si } \delta \geq 2 \lambda .
\end{aligned}
$$

Posons

$$
\begin{aligned}
& \sigma_{L}^{\delta}(\cos \theta, \cos \phi)=c_{\lambda} \int_{0}^{\pi} \sigma_{L}^{\delta}(\cos \Omega) \sin ^{2 \lambda-1} w d w ; \\
& \tau_{L}^{\delta}(\cos \theta, \cos \phi)=c_{\lambda} \int_{0}^{\pi} \tau_{L}^{\delta}(\cos \Omega) \sin ^{2 \lambda-1} w d w .
\end{aligned}
$$

Lemme (6.2). Il existe une constante $A$ indépendante de $L$ telle que

(i) $\left|\sigma_{L}^{\delta}(\cos \theta, \cos \phi)\right| \leq A L^{\lambda-\delta}\left(L^{-2}+|\theta-\phi|^{2}\right)^{-(\lambda-\delta-1) / 2}$ si $\delta \leq \lambda+1$;

(ii) $\left|\sigma_{L}^{\delta}(\cos \theta, \cos \phi)\right| \leq A L^{-1}\left(L^{-2}+|\theta-\phi|^{2}\right)^{-\lambda-1}$ si $\delta \geq \lambda+1$;

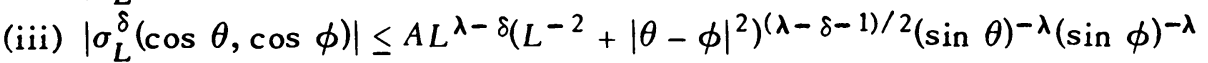
si $\lambda-1<\delta \leq \lambda+1$;

(iv) $\left|\sigma_{L}^{\delta}(\cos \theta, \cos \phi)\right| \leq A L^{-1}\left(L^{-2}+|\theta-\phi|^{2}\right)^{-1}(\sin \theta)^{-\lambda}(\sin \phi)^{-\lambda}$ si $\delta \geq \lambda+1$

(v) $\left|\tau_{L}^{\delta}(\cos \theta, \cos \phi)\right| \leq A|\pi-\theta-\phi|^{-2 \lambda+\delta}$ si $\delta \leq 2 \lambda$;

(vi) $\left|r_{L}^{\delta}(\cos \theta, \cos \phi)\right| \leq A$ si $\delta \geq 2 \lambda$.

Nous allons donner la démonstration de (i) et (iii). Les majorations (ii) et (iv) sont des cas particuliers des majorations (i) et (iii), les majorations (v) et (vi) s'obtiennent de la même manière.

Soit donc $\delta \leq \lambda+1 . \Omega^{2} \geq 4 \sin ^{2} \Omega / 2=2(1-\cos \Omega)$, donc

$$
\begin{aligned}
& \sigma_{L}^{\delta}(\cos \Omega) \leq A L^{\lambda-\delta}\left(L^{-2}+1-\cos \Omega\right)^{(-\lambda-\delta-1) / 2} \\
& \quad \leq A L^{\lambda-\delta}\left(L^{-2}+1-\cos (\theta-\phi)+(1-\cos w) \sin \theta \sin \phi\right)^{(-\lambda-\delta-1) / 2} \\
& \quad \leq A L^{\lambda-\delta}\left(L^{-2}+|\theta-\phi|^{2}+w^{2} \sin \theta \sin \phi\right)^{(-\lambda-\delta-1) / 2}
\end{aligned}
$$

puisque $0<w / 2<\pi / 2,0<|\theta-\phi| / 2<\pi / 2$.

$$
\begin{aligned}
& \sigma_{L}^{\delta}(\cos \theta, \cos \phi)=c_{\lambda} \int_{0}^{\pi / 2} \cdots+c_{\lambda} \int_{\pi / 2}^{\pi} \cdots=I_{1}+I_{2} \text {. } \\
& \mathrm{I}_{1} \leq A L^{\lambda-\delta} \int_{0}^{\pi / 2}\left(L^{-2}+|\theta-\phi|^{2}+w^{2} \sin \theta \sin \phi\right)^{(-\lambda-\delta-1) / 2} w^{2 \lambda-1} d w \\
& =A L^{\lambda-\delta}(\sin \theta)^{-\lambda}(\sin \phi)^{-\lambda}\left(L^{-2}+|\theta-\phi|^{2}\right)^{(\lambda-\delta-1) / 2} \\
& \int_{0}^{T}\left(1+w^{2}\right)^{(-\lambda-\delta-1) / 2} w^{2 \lambda-1} d w \\
& \text { où } T=\frac{\pi}{2}\left(\frac{\sin \theta \sin \Phi}{L^{-2}+|\theta-\Phi|^{2}}\right) \text {. }
\end{aligned}
$$


Lorsque $\delta>\lambda-1, \int_{0}^{\infty}\left(1+w^{2}\right)^{(-\lambda-\delta-1) / 2} w^{2 \lambda-1} d w<\infty$, d'où la majoration de type (iii) pour $I_{1}$. D'autre part $\int_{0}^{T}\left(1+w^{2}\right)^{(-\lambda-\delta-1) / 2} w^{2 \lambda-1} d w \leq A T^{2 \lambda}$, d'où la majoration de type (i) pour $I_{1}$. L'intégrale $I_{2}$ se traite de manière analogue.

Lemme (6.3). Soit $\delta>\lambda_{0}$ Il existe une constante $A$, indépendante de f, telle que $\sup _{L}\left|S_{L}^{\delta} f(\theta)\right| \leq A(M f(\theta)+M f(\pi-\theta))$.

Nous allons montrer que $\left|\int_{0}^{\pi} \sigma_{L}^{\delta}(\cos \theta, \cos \phi) f(\phi) d \phi\right| \leq A M f(\theta)$, tandis que $\left|\int_{0}^{\pi} \tau_{L}^{\delta}(\cos \theta, \cos \phi) f(\phi) d \phi\right| \leq A M f(\pi-\theta)$. Posons

$$
M^{*} f(\theta)=\sup _{b \neq 0 ; 0 \leq \theta+b \leq \pi} \frac{\int_{\theta}^{\theta+b}|f(\phi)| d m_{2 \lambda}(\phi)}{\int_{\theta}^{\theta+b} d m_{2 \lambda}(\phi)} .
$$

Un calcul élémentaire permet de montrer que $M^{*} f$ est, à une constante indépendante de $f$ près, majorée par $M f$. Or, pour montrer l'inéga lité $\left|\int_{0}^{\pi} \sigma_{L}^{\delta}(\cos \theta, \cos \phi) f(\phi) d \phi\right|$ $\leq A m^{*} f(\theta)$, il suffit de montrer l'existence d'une fonction positive $k_{L}(\phi)$ qui majore $\sigma_{L}^{\delta}(\cos \theta, \cos \phi)$, soit croissante dans $[0, \theta[$, décroissante dans $] \theta, \pi]$, et telle que

$$
\begin{gathered}
k_{L}(0) \leq A\left(\int_{0}^{\theta} \sin ^{2 \lambda} \phi d \phi\right)^{-1}, \quad k_{L}(\pi) \leq A\left(\int_{\theta}^{\pi} \sin ^{2 \lambda} \phi d \phi\right)^{-1}, \\
\int k_{L}(\phi) \sin ^{2 \lambda} \phi d \phi \leq A,
\end{gathered}
$$

comme le montre une intégration par parties élémentaire, semblable à celle qui a été faite dans le $\$ 3$. Supposons $\delta \leq \lambda+1, \theta \leq \pi / 2$, le cas général s'en déduisant immédiatement. Si $\theta \leq 2 / L$, on peut prendre $k_{L}(\phi)=A L^{\lambda-\delta}\left(L^{-2}+|\theta-\phi|^{2}\right)^{(-\lambda-\delta-1) / 2}$. Si $\theta>2 / L$, on peut prendre

$$
\begin{aligned}
& k_{L}(\phi)=A L^{\lambda-\delta\left(L^{-2}+|\theta-\phi|^{2}\right)^{(\lambda-\delta-1) / 2}(\sin \theta)^{-2 \lambda}} \text { si } \theta / 2 \leq \phi \leq 3 \theta / 2, \\
& k_{L}(\phi)=A^{\prime} L^{\lambda-\delta}|\theta-\phi|^{-\lambda-\delta-1} \text { sinon. }
\end{aligned}
$$

L'inégalité $\left|\int_{0}^{\pi} \tau_{L}^{\delta}(\cos \theta, \cos \phi) f(\phi) d \phi\right| \leq A M^{*} f(\pi-\theta)$ s'obtient de manière plus élémentaire encore.

Théorème (6.4). Soit $\delta \geq 0$. Si $p>1$ et si $|1 / p-1 / 2| \leq(\delta+1 / 2) /(2 \lambda+1)$, il existe une constante $A_{p}$ telle que, pour toute fonction $f \in L^{p}\left(d m_{2 \lambda}\right),\left\|\sup _{L}\left|S_{L}^{\delta} f\right|\right\|_{p}$ $\leq A_{p}\|f\|_{p}$. Sous les mêmes conditions, les moyennes de Cesàro d'ordre $\delta$ de $f$ convergent vers $f$ presque partout.

Lorsque $\delta=0$, ce résultat est dû à Gilbert [10]. Lorsque $\delta>\lambda$, il est conséquence du Lemme (6.3). Le résultat intermédiaire s'obtient par interpolation, de la même manière que le Corollaire (3.4).

Théorème (6.5). Soit $\delta \geq 0, p>1$. Si $-(2 \lambda+1) / p+\sup (0, \lambda-\delta)<\alpha<$ $(2 \lambda+1) / p^{\prime}-\sup (0, \lambda-\delta)$ il existe une constante $A_{p, a}$ telle que, pour toute suite de fonctions $f_{k} \in L^{p}\left(d m_{2 \lambda}\right)$ et tout choix d'indices $L_{k}$, 


$$
\left\|\left(\sum\left|S_{L_{k}}^{\delta} f_{k}\right|^{2}\right)^{1 / 2}\right\|_{p, a} \leq A_{p a}\left\|\left(\sum\left|f_{k}\right|^{2}\right)^{1 / 2}\right\|_{p, a}
$$

Lorsque $\delta=0$, ce résultat est dû à Muckenhoupt et Stein [17]. Lorsque $\delta>\lambda$, il résulte des Lemmes (6.1) et (6.3). Les cas intermédiaires sont obtenus en interpolant entre les espaces de fonctions

$$
\begin{aligned}
& L_{a_{0}}^{p}\left(d m_{2 \lambda}\right)=\left\{f ; f(\theta) \sin ^{a_{0}} \theta \in L^{p}\left(d m_{2 \lambda}\right)\right\}, \quad(-2 \lambda+1) / p<a_{0}<(2 \lambda+1) / p^{\prime}, \\
& \text { et }{ }_{a_{1}}^{p}\left(d m_{2 \lambda}\right)=\left\{f ; f(\theta) \sin ^{1} \theta \in L^{p}\left(d m_{2 \lambda}\right)\right\}, \quad(-2 \lambda+1) / p+\lambda<\alpha_{1}<(2 \lambda+1) / p^{\prime}-\lambda,
\end{aligned}
$$
par la méthode des familles analytiques d'opérateurs: on sait que, si $\delta>\lambda$,

$$
\begin{aligned}
& \left\|\left(\sum\left|S_{L}^{\delta+i y} f_{k}\right|^{2}\right)^{1 / 2}\right\|_{p, a_{0}} \leq A_{p a_{0}} e^{c y}\left\|\left(\sum\left|f_{k}\right|^{2}\right)^{1 / 2}\right\|_{p, a_{0}} \\
& \left\|\left(\sum\left|S_{L}^{\delta+i y} f_{k}\right|^{2}\right)^{1 / 2}\right\|_{p, a_{1}} \leq A_{p a_{1}} e^{c y}\left\|\left(\sum\left|f_{k}\right|^{2}\right)^{1 / 2}\right\|_{p, a_{1}} \text { si } \delta>0 .
\end{aligned}
$$

Il suffit alors d'utiliser le fait bien connu que l'interpolé $\left(L_{a_{0}}^{p}\left(d m_{2 \lambda}\right), L_{a_{1}}^{p}\left(d m_{2 \lambda}\right)\right)_{\theta}$, au sens de Calderón, est $L_{\alpha_{0} \theta+a_{1}(1-\theta)}^{p}\left(d m_{2 \lambda}\right)$.

Théorème (6.6). Soit $p>1, k$ un entier supérieur ou égal à 1. Si la suite $\mu_{n}$ satisfait aux conditions:

$$
\begin{aligned}
& \left(A_{0}\right) \sup \left|\mu_{n}\right|<M, \\
& \left(A_{k}\right) \sup 2^{(k-1) n} \sum_{j=2^{n}}^{2^{n+1}}\left|\Delta^{k} \mu_{j}\right|<M,
\end{aligned}
$$

alors, il existe une constante $A_{p}, a$, indépendante de $\mu_{n}$ telle que, pour toute fonction $\sum a_{n} P_{n}^{\lambda}(\cos \theta) \in L_{a}^{p}\left(d m_{2 \lambda}\right)$, où:

$$
\begin{gathered}
-(2 \lambda+1) / p+\sup (0, \lambda-k+1)<a<(2 \lambda+1) / p^{\prime}-\sup (0, \lambda-k+1) \\
\left\|\sum \mu_{n} a_{n} P_{n}^{\lambda}(\cos \theta)\right\|_{p, a} \leq M A_{p, a}\|f\|_{p, a} .
\end{gathered}
$$

Lorsque $k=1$, ce théorème est dû à Muckenhoupt et Stein [17]. Lorsque $k \leq \lambda+1$, il a été démontré par une méthode entièrement différente dans [2].

Dans tous les cas, c'est une conséquence des inégalités:

$$
\|g(f)\|_{p, a} \leq A_{p}\|f\|_{p, a} \quad\|f\|_{p, a} \leq A_{p}\left(\left|a_{0}\right|+\|g(f)\|_{p, a}\right),
$$

où $g(f)=\left(\int_{0}^{1}(1-r)\left|\partial P_{r}(f) / \partial r\right|^{2} d r\right)^{1 / 2}$ et $P_{r} f(\theta)=\sum a_{n} r^{n} P_{n}^{\lambda}(\cos \theta)$, (voir [17]), du Théorème (6.5), et du Théorème (4.7), ou du moins de sa transposition dans le cadre des polynômes ultrasphériques.

7. Généralisations diverses du théorème de multiplicateurs (4.9). Nous avons déjà signalé dans l'introduction que la méthode utilisée au $\$ 4$ se généralise à bien d'autres situatiòns dès lors que l'on sait pour quelles valeurs de $\delta$ une inégalité du type:

$$
\left\|\left(\sum\left|S_{n_{k}}^{\delta} f_{k}\right|^{2}\right)^{1 / 2}\right\|_{p} \leq A_{p}\left\|\left(\sum\left|f_{k}\right|^{2}\right)^{1 / 2}\right\|_{p}
$$


est réalisée. C'est le cas, en particulier, non seulement pour les sphères, mais pour tous les espaces symétriques compacts de rang 1.

La liste exhaustive des espaces symétriques compacts de rang 1 est donnée dans [9]. Ce sont $\Sigma_{n}=S O(n+1) / S O(n), P_{n}(\mathbf{R})=S O(n+1) / O(n), P_{n}(\mathbf{C})=$ $S U(l+1) / S(U(l) \times U(1))($ avec $l=n / 2 \geq 2), P_{n}(H)=S p(l+1) / S_{p}(l) \times S_{p}(1)$ (avec $l=n / 4 \geq 2), P_{16}=F_{4(-52)} / S O(9)$. Soit $M=G / K$ un de ces espaces, $1=e K$ (e élément neutre de $G$ ). Les géodésiques ont toutes même longueur, que nous poserons égale à $2 D$, et les fonctions zonales peuvent entre identifiées à des fonctions sur $[0, D]$. L'application exponentielle au pôle 1 permet de définir un système de coordonnées polaires sur $M,(\theta, u)$, avec $0 \leq \theta \leq D$ qui représente la distance à 1 , et $u \in \Sigma_{n-1}$. La mesure invariante sur $M$ est donnée par $\sin ^{p} \lambda \theta \sin ^{q} 2 \lambda \theta d \theta d u$, à une constante près, $p, q$ et $\lambda$ étant égaux à :

\begin{tabular}{c|c|c|c|c|c}
$M$ & $\Sigma_{n}$ & $P_{n}(\mathbf{R})$ & $P_{n}(\mathbf{C})$ & $P_{n}(H)$ & $P_{16}$ \\
\hline$p$ & 0 & 0 & $n-2$ & $n-4$ & 8 \\
\hline$q$ & $n-1$ & $n-1$ & 1 & 3 & 7 \\
\hline$\lambda$ & $\pi / 2 D$ & $\pi / 4 D$ & $\pi / 2 D$ & $\pi / 2 D$ & $\pi / 2 D$
\end{tabular}

Posons $\alpha=(p+q-1) / 2, \beta=(q-1) / 2$. L'opérateur de Laplace-Beltrami sur $M$ admet pour valeurs propres $k(k+\alpha+\beta+1)$, où $k=0,1,2, \ldots$ sauf lorsque $M=P_{n}(\mathbf{R})$, où les seules valeurs admises pour $k$ sont $k=0,2,4, \ldots$, et les fonctions propres zonales sont, à une constante près, $P_{k}^{a, \beta}(\cos 2 \lambda \theta)$.

Considérons tout d'abord le cas où $M$ n'est pas $P_{n}(\mathbf{R})$. Appelons $\mathcal{H}_{k}$ le sous-espace propre relatif à la valeur propre $k(k+\alpha+\beta+1), H_{k} f$ la projection de $f$ sur $\mathcal{H}_{k}$. La moyenne de Cesàro $S_{L}^{\delta} f$ est définie par

$$
j_{L}^{\delta} f=\left(A_{L}^{\delta}\right)^{-1} \sum_{l=0}^{L} A_{L-l}^{\delta} H_{l} f,
$$

et, comme dans le cas de $\Sigma_{n}$, est donnée par la convolution avec la fonction zonale:

$$
s_{L}^{\delta}\left(\cos \frac{\pi \theta}{D}\right)=\left(A_{L}^{\delta}\right)^{-1} \sum_{l=0}^{L} A_{L-l}^{\delta}\left\|P_{l}^{a, \beta}\right\|_{2}^{-2} P_{l}^{a, \beta}(1) P_{l}^{a, \beta}\left(\cos \frac{\pi \theta}{D}\right) .
$$

Les majorations obtenues au $\S 2$ :

$$
\begin{aligned}
& \left|s_{L}^{\delta}(\cos \theta)\right| \leq A L^{2 a+2}, \\
& \left|S_{L}^{\delta}(\cos \theta)\right| \leq A L^{a+1 / 2-\delta}|\theta|-a-\delta-3 / 2, \quad \pi / L<\theta<\pi / 2, \\
& \left|s_{L}^{\delta}(\cos \theta)\right| \leq A L^{a+\beta+1-\delta}, \quad \pi / 2<\theta<\pi, \\
& \left|s_{L}^{\delta}(\cos \theta)\right| \leq A L^{a+1 / 2-\delta}|\pi-\theta|^{-1 / 2}, \quad \pi / 2<\theta<\pi-\pi / L,
\end{aligned}
$$


lorsque $\delta<a+3 / 2$, permettent, là encore, d'obtenir la majoration (pour $\delta>a+1 / 2)(8)$ :

$$
\sup _{L}\left|S_{L}^{\delta} f\right| \leq A M f+K *|f|
$$

avec $K \in L^{1}(M)$. Les analogues du Théorème (3.6) et du Corollaire (3.7) s'en déduisent immédiatement.

D'autre part, l'intégrale de Poisson de $f, P_{r} f=\Sigma_{r} l_{l} f$ est donnée, là encore, par la convolution avec le noyau de Poisson:

$$
P_{r}\left(\cos \frac{\pi \theta}{D}\right)=\sum r^{l}\left\|P_{l}^{a, \beta}\right\|_{2}^{-2} P_{l}^{\alpha, \beta}(1) P_{l}^{a, \beta}\left(\cos \frac{\pi \theta}{D}\right) .
$$

Il résulte de [22] que $P_{r}$ est positif, donc de norme 1 dans $L^{1}(M)$, et donc que $P$, définit un semi-groupe satisfaisant aux propriétés requises pour l'existence d'inégalités $L^{p}$ pour la fonction de Paley-Littlewood correspondante [19]: si $g(f)=\left(\int_{0}^{1}(1-r)\left|\partial\left(P_{r} f\right) / \partial r\right|^{2} d r\right)^{1 / 2},\|g(f)\|_{p} \leq A_{p}\|f\|_{p}$ et, si $H_{0} f=0,\|f\|_{p} \leq$ $A_{p}\|g(f)\|_{p}$. On obtient ainsi l'analogue du théorème de multiplicateurs (4.9).

Considérons maintenant le cas où $M$ est $P_{n}(\mathbf{R}): H_{k}$ désignera le sous-espace propre relatif à la valeur propre $2 k(2 k+\alpha+\beta+1)$, et plutôt que les moyennes de Cesàro, nous définirons:

$$
\sigma_{L}^{\delta} f=\left(A_{2 L}^{\delta}\right)^{-1} \sum_{l=0}^{L} A_{2 L-2 l}^{\delta} H_{l} f
$$

$\sigma_{L}^{\delta} f$ est donné par la convolution avec la fonction zonale:

$$
\begin{aligned}
& \sigma_{L}^{\delta}\left(\cos \frac{\pi \theta}{2 D}\right)=\left(A_{2 L}^{\delta}\right)^{-1} \sum_{l=0}^{L} A_{2 L-2 l}^{\delta}\left\|P_{2 l}^{a, \beta}\right\|_{2}^{-2} P_{2 l}^{a, \beta}(1) P_{l}^{a, \beta}\left(\cos \frac{\pi \theta}{2 D}\right), \\
& \sigma_{L}^{\delta}\left(\cos \frac{\pi \theta}{2 D}\right)=\frac{1}{2}\left[s_{2 L}^{\delta}\left(\cos \frac{\pi \theta}{2 D}\right)+s_{2 L}^{\delta}\left(\cos \left(\frac{\pi}{2 D}(2 D-\theta)\right)\right)\right],
\end{aligned}
$$

d'où la majoration

$$
\begin{gathered}
\left|\sigma_{L}^{\delta}\left(\cos \frac{\pi \theta}{2 D}\right)\right| \leq A L^{2 a+2}, \\
\sigma_{L}^{\delta}\left(\cos \frac{\pi \theta}{2 D}\right) \leq A L^{a+1 / 2-\delta}|\theta|^{-a-\delta-3 / 2}, \quad \frac{\pi \theta}{D}>\frac{\pi}{L} .
\end{gathered}
$$

Donc $\sup _{L}\left|\sigma_{L}^{\delta} f\right| \leq A M f$ si $\delta>a+1 / 2$, et pour toutes suites $f_{k}$, $L_{k}$, et $p>1$

avec $\delta=a+1 / 2$.

$$
\left\|\left(\sum\left|\sigma_{L_{k}}^{\delta} f_{k}\right|^{2}\right)^{1 / 2}\right\|_{p} \leq A_{p}\left\|\left(\sum\left|f_{k}\right|^{2}\right)^{1 / 2}\right\|_{p}
$$

De même, l'intégrale de Poisson de $f$ est donnée par la convolution avec le noyau

(8) Remarquer que $a+1 / 2=(n-1) / 2$ où $n$ est la dimension de $M$. 


$$
\begin{aligned}
P_{r}\left(\cos \frac{\pi \theta}{2 D}\right) & =\sum r^{l}\left\|P_{2 l}^{a,}\right\|_{2}^{-2} P_{2 l}^{a, a}(1) P_{2 l}^{a, a}\left(\cos \frac{\pi \theta}{2 L}\right) \\
& =\frac{1}{2}\left(\frac{1-r}{(1-2 \sqrt{r} \cos (\pi \theta / 2 D)+r)^{(a+3) / 2}}+\frac{1-r}{(1+2 \sqrt{r} \cos (\pi \theta / 2 D)+r)^{(a+3) / 2}}\right)
\end{aligned}
$$

qui est positif, uniformément de norme 1 dans $L^{1}(M)$. D'où les inégalités pour la fonction de Paley-Littlewood.

Le théorème de multiplicateurs de type (4.9) s'en déduit aisément, à condition d'avoir montré que les lemmes combinatoires (4.4), (4.5) et (4.8) sont encore vrais lorsque les moyennes de Cesàro $s_{L}^{\delta}=\left(A_{L}^{\delta}\right)^{-1} \Sigma_{l=0}^{L} A_{L-l}^{\delta} u_{l}$ sont remplacées par les moyennes $\left(A_{2 L}^{\delta}\right)^{-1} \Sigma_{l=0}^{L} A_{2 L-2 l}^{\delta} u_{l}$. Mais il suffit de remarquer que cette dernière quantité peut être obtenue comme la demie sommes des moyennes de Cesàro des suites $u_{0}, u_{1}, u_{1}, \ldots, u_{l}, u_{l}, \ldots$, et $u_{0},-u_{1}, u_{1}$, $\cdots,-u_{l}, u_{l}$. On peut finalement énoncer le théorème suivant:

Théorème (7.1). Soit $M$ un espace symétrique compact de rang 1 de dimension $n$. Il existe une constante $A_{p}$, pour tout $p>1$, telle que quelle que soit la suite $\mu_{j}$ satisfaisant aux conditions:

$$
\begin{aligned}
& \left(A_{0}\right) \sup \left|\mu_{j}\right| \leq M<\infty, \\
& \left(A_{k}\right) \sup _{j} 2^{j(k-1)} \Sigma_{l=2^{j}}^{2^{j+1}}\left|\Delta^{k} \mu_{l}\right| \leq M<\infty,
\end{aligned}
$$

avec $k=(n+1) / 2$ si $n$ est impair, $k=(n+2) / 2$ si $n$ est pair, on ait l'inégalité

$$
\left\|\sum \mu_{j} H_{j} f\right\|_{p} \leq A_{p} M\|f\|_{p}
$$

pour toute fonction $f \in L^{p}(M)$.

Nous n'écrirons pas l'analogue de (4.9) (2) et de (4.10), qui sont tout aussi valables.( $(9)$

Montrons enfin que la méthode s'applique encore dans le cas de variétés riemanniennes compactes. Soit donc $(\Re, g)$ une variété riemannienne de dimension $n, \Delta$ son Laplacien et $I=\int_{0}^{+\infty} d E_{\lambda}$ la résolution spectrale de l'identité associée. On peut, comme dans [19], définir une fonction de Littlewood-Paley à l'aide du noyau de la chaleur: on pose $T^{t} f(x)=\int_{0}^{+\infty} e^{-\lambda t} d E_{\lambda} f$, et $g(f)=\left(\int_{0}^{+\infty} t\left|\partial T^{t} f / \partial t\right|^{2} d t\right)^{1 / 2}$; on a alors les inégalités $\|g(f)\|_{p} \leq A_{p}\|f\|_{p}$, et $\|f\|_{p} \leq\|g(f)\|_{p}$, cette dernière dès lors que $\int f=0(1<p<+\infty)$. Nous utiliserons ici les sommes de Riesz qui sont définies comme suit:

$$
S_{R}^{\delta} f=\int_{0}^{R}\left(1-\frac{\lambda}{R}\right)^{\delta} d E_{\lambda} f
$$

Hörmander a démontré que si $\delta \geq n-1$, on a l'inéga lité (voir [12], [13]) $\sup _{R}\left|S_{R}^{\delta} f\right| \leq C$. Mf, où $M f$ est la fonction maximale au sens de Hardy-Littlewood, $M f(x)=\sup _{r}(1 /|B(x, r)|) \int_{B(x, r)}|f(y)| d y$. Cette inégalité, jointe au résultat de

(9) On remarquera que les résultats de localisation qu'on peut, comme dans le cas de la sphère, déduire des inégalités obtenues dépendent fortement de l'espace symétrique choisi et non plus uniquement de sa dimension. 
Fefferman-Stein permet alors d'obtenir "l'inégalité de Zygmund":

$$
\left\|\left(\sum_{i}\left|S_{R_{i}}^{\delta} f_{i}(x)\right|^{2}\right)^{1 / 2}\right\|_{p} \leq A_{p}\left\|\left(\sum_{i}\left|f_{i}(x)\right|^{2}\right)^{1 / 2}\right\|_{p} \quad(1<p<+\infty) .
$$

On peut alors déduire de ce résultat un théorème de multiplicateurs, comme on l'a fait au $\$ 4$.

Théorème (7.2). Soit $m(\lambda)$ une fonction $n$ fois dérivable sur $[0,+\infty)$, et satisfaisant aux conditions:

$$
\begin{aligned}
& \left(A_{0}\right) \sup _{\lambda}|m(\lambda)| \leq M<+\infty, \\
& \left(A_{n}\right) \sup _{\mathbf{\Lambda}}(1 / \Lambda) \int_{0}^{\Lambda}\left|\lambda^{n}(d / d \lambda)^{n} m(\lambda)\right| d \lambda \leq M<+\infty,
\end{aligned}
$$

alors

$$
\left\|\int_{0}^{+\infty} m(\lambda) d E_{\lambda} f\right\|_{p} \leq C_{p} \cdot\|f\|_{p}
$$

La démonstration suit celle du Théorème (4.7), avec les variantes qu'impose le recours aux sommes de Riesz. On introduit d'abord la fonction de LittlewoodPaley $g_{\delta}(f)=\left(\int_{0}^{+\infty}\left|S_{R}^{\delta+1} f-S_{R}^{\delta} f\right|^{2} d R / R\right)^{1 / 2}$. On montre comme précédemment que $g(f) \leq C \cdot g_{\delta}(f)$, puis, ici seulement pour $\delta$ entier que $g_{\delta}^{x}(f)=$ $\left(\int_{0}^{+\infty}\left|S_{R}^{\delta+1} f-S_{R}^{\delta} f\right|^{2} \nu(R) d R / R\right)^{1 / 2}$, où $\int_{0}^{R} \nu(r) d r \leq M \cdot R$, satisfait, lorsque $\delta \geq n-1$ l'inégalité $\left\|g_{\delta}^{*}(f)\right\|_{p} \leq C_{p}\|g(f)\|_{p}$. Pour ceci, nous avons besoin d'un résultat auxiliaire.

Lemme (7.3). Soit $\mu$ une mesure sur $[0,+\infty)$; posons $S_{R}^{\delta}=\int_{0}^{R}(1-\lambda / R)^{\delta} d \mu(\lambda)$ et $\sigma_{R}^{\delta}=\int_{0}^{R}(1-\lambda / R)^{\delta} e^{-t \lambda} d \mu(\lambda)$, avec $0<t \leq 1 / R$; alors $s_{R}^{\delta}=e^{R t} \sigma_{R}^{\delta}+$ $\int_{0}^{R} \sigma_{\lambda}^{\delta} A(\lambda, R) d \lambda$, ò̀ $A(\lambda, R)=O(t)$.

En effet,

$$
\begin{aligned}
s_{R}^{\delta}= & \int_{0}^{R}(1-\lambda / R)^{\delta} e^{t \lambda} e^{-t \lambda} d \mu(\lambda)=e^{R t} \int_{0}^{R}(1-\lambda / R)^{\delta} e^{-t \lambda} d \mu(\lambda) \\
& +e^{R t} \int_{0}^{R}(1-\lambda / R)^{\delta} e^{-\lambda t}\left(e^{-t(R-\lambda)}-1\right) d \mu(\lambda)
\end{aligned}
$$

Mais

$$
\begin{aligned}
& \int_{0}^{R}(1-\lambda / R)^{\delta}\left(e^{-t(R-\lambda)}-1\right) \cdot e^{-\lambda t} d \mu(\lambda) \\
&=-R^{-\delta} \int_{0}^{R} \frac{d}{d \lambda}\left\{(R-\lambda)^{\delta}\left(e^{-t(R-\lambda)}-1\right)\right\}\left(\int_{0}^{\lambda} e^{-t \tau} d \mu(\tau)\right) d \lambda .
\end{aligned}
$$

Intégrant à nouveau $\delta$ fois par parties, il vient 


$$
\begin{aligned}
\int_{0}^{R}\left(1-\frac{\lambda}{R}\right)^{\delta}\left(e^{-t(R-\lambda)}-1\right) e^{-\lambda t} d \mu(\lambda) \\
\quad=(-1)^{\delta+1} R^{-\delta} \int_{0}^{R}\left(\frac{d}{d \lambda}\right)^{\delta+1}\left\{(R-\lambda)^{\delta} e^{-t(R-\lambda)}-1\right\}\left(\int_{0}^{\lambda}(\lambda-\tau)^{\delta} e^{-t \tau} d \mu(\tau)\right) d \lambda \\
\quad=(-1)^{\delta+1} \int_{0}^{R}\left(\frac{\lambda}{R}\right)^{\delta}\left(\frac{d}{d \lambda}\right)^{\delta+1}\left\{(R-\lambda)^{\delta}\left(e^{-t(R-\lambda)}-1\right\} \cdot \sigma_{\lambda}^{\delta} \cdot d \lambda\right.
\end{aligned}
$$

Développant par la règle de Leibniz, on voit apparaitre des termes

$$
(\lambda / R)^{\delta} \cdot(R-\lambda)^{\delta-b} e^{-t(R-\lambda)} t^{\delta+1-b}=t \cdot e^{-t(R-\lambda)}((R-\lambda) \cdot t)^{\delta-b} \cdot(\lambda / R)^{\delta} \leq t .
$$

D'où le lemme.

Venons à la démonstration du résultat sur la fonction $g_{\delta}^{*}$ : d'abord

$$
S_{R}^{\delta+1} f-S_{R}^{\delta} f=-\frac{1}{R} \int_{0}^{R}\left(1-\frac{\lambda}{R}\right)^{\delta} \lambda d E_{\lambda} f
$$

notons ensuite $f_{t}=T^{t} f ;$ alors

$$
S_{R}^{\delta+1} f_{t}-S_{R}^{\delta+1} f_{t}=-\frac{1}{R} \int_{0}^{R}\left(1-\frac{\lambda}{R}\right)^{\delta} \lambda e^{-\lambda t} d E_{\lambda} f
$$

Grâce au lemme, et à l'inégalité de Schwarz, on en déduit:

$$
\left|S_{R}^{\delta+1} f-S_{R}^{\delta} f\right|^{2} \leq A\left(\left|S_{R}^{\delta+1} f_{t}-S_{R}^{\delta} f_{t}\right|^{2}+R t^{2} \int_{0}^{R}\left|S_{\lambda}^{\delta+1} f_{t}-S_{\lambda}^{\delta} f_{t}\right|^{2} d \lambda\right)
$$

dès lors que $t \leq 1 / R$; raisonnant comme au $\$ 4$, on peut toujours supposer que $R \leq \int_{0}^{R} \nu(r) d r \leq \overline{2} R$, de sorte qu'on choisira $1 / t(R)=\int_{0}^{R} \nu(r) d r$; montrons d'abord que

$$
\begin{gathered}
\left\|\left(\int_{0}^{+\infty}\left|S_{R}^{\delta+1} f_{t(R)}-S_{R}^{\delta} f_{t(R)}\right|^{2} \nu(R) \frac{d R}{R}\right)^{1 / 2}\right\|_{p} \leq A_{p}\|g(f)\|_{p} \\
S_{R}^{\delta+1} f_{t}-S_{R}^{\delta} f_{t}=(1 / R) S_{R}^{\delta}\left(\frac{\partial}{\partial t} T^{t} f\right)
\end{gathered}
$$

on utilise maintenant "l'inéga lité de Zygmund" convenablement généralisée, d'où

$$
\begin{aligned}
\|\left(\int_{0}^{+\infty} \mid S_{R}^{\delta+1} f_{t(R)}-\right. & \left.\left.S_{R}^{\delta} f_{t(R)}\right|^{2} \nu(R) \frac{d R}{R}\right)^{1 / 2} \|_{p} \\
& \leq A_{p}\left\|\left(\int_{0}^{+\infty} \frac{1}{R^{2}}\left|\left(\frac{\partial}{\partial t} T^{t} f\right)_{t=t(R)}\right|^{2} \nu(R) \frac{d R}{R}\right)^{1 / 2}\right\|_{p}
\end{aligned}
$$

Il ne reste plus qu'à faire le changement de variable $R \rightarrow t=t(R)$ pour reconnaitre dans le second membre $\|g(f)\|_{p}$. Ensuite 


$$
\begin{aligned}
\|\left(\int_{0}^{+\infty} R \cdot t(R)^{2} \int_{0}^{R}\right. & \left.\left|S_{\lambda}^{\delta+1} f_{t(R)}-S_{\lambda}^{\delta} f_{t(R)}\right|^{2} d \lambda \cdot \nu(R) \frac{d R}{R}\right)^{1 / 2} \|_{p} \\
& \leq A_{p}\left\|\left(\int_{0}^{+\infty} R t(R)^{2} \cdot \int_{0}^{R}\left|\left(\frac{\partial}{\partial t} T^{t} f\right)_{t=t(R)}\right|^{2} \frac{d \lambda}{\lambda^{2}} \cdot \nu(R) \frac{d R}{R}\right)^{1 / 2}\right\|_{p} \\
& \leq A_{p}\left\|\left(\int_{0}^{+\infty} R \cdot t(R)^{2} \cdot \frac{1}{R} \frac{\nu(R)}{R}\left|\left(\frac{\partial}{\partial t} T^{t} f\right)_{t=t(R)}\right|^{2} d R\right)^{1 / 2}\right\|_{p} .
\end{aligned}
$$

On fait maintenant le changement de variable $R \rightarrow t$, et on tient compte de ce que $t(R) \geq 1 / R$; d'où encore la majoration par $\|g(f)\|_{p}$.

La suite de la démonstration est alors tout à fait similaire à celle du Théorème (4.7), l'analogue du Lemme (4.8) se démontrant comme le Lemme (7.3).

Remarque. L'inégalité démontrée par Hörmander admet effectivement $\delta=n-1$ pour indice critique (cf. le cas des sphères). Il serait intéressant de rechercher une inégalité analogue à celle du Théorème (3.1) dans le cas d'une variété compacte quelconque. Dans le cas des groupes de Lie compacts, la démonstration d'une telle inégalité fera l'objet d'une publication ultérieure de l'un des deux auteurs. Les travaux de N. Weiss [24] laissaient prévoir que le Théorème (7.2) pouvait être amélioré dans ce cas.

\section{BI BLIOGRAPHIE}

1. R. A skey and I. I. Hirschman, Jro, Mean summability for ultraspherical polynomials, Math. Scand. 12 (1963), 167-177. MR 29 \# 1497.

2. A. Bonami, Multiplicateurs des séries ultrasphériques, C. R. Acad. Sci. Paris Sér. A-B 273 (1971), A148-A150。

3. J.-L. Clerc, Fonctions de Paley-Littlewood sur SU(2) attachées aux sommes de Riesz, C. R. Acad. Sci。 Paris Sér。A-B 272 (1971), A1697-A1699. MR 43 \#4962.

4. - Les sommes partielles de la décomposition en harmoniques sphériques ne convergent pas dans $L^{p}(p \neq 2)$, C. R. Acad. Sci. Paris 274 (1972), 59-61.

5. R. Coifman et G. Weiss, Analyse harmonique non commutative sur certains espaces homogènes, Lecture Notes in Math., no. 242, Springer-Verlag, Berlin and New York, 1971.

6. C. Fefferman, Inequalities for strongly singular convolution operators, Acta Math, 124 (1970), 9-36. MR 41 \#2468.

7. - The multiplier problem for the ball, Ann. of Math. (2) 94 (1971), 330-336.

8. C. Fefferman and E. Stein, Some maximal inequalities, Amer. J. Math. 93 (1971), $107-115$.

9. R. Gangolli, Positive definite kernels on homogeneous spaces and certain stochastic processes related to Lévy's Brownian motion of several parameters, Ann. Inst. H. Poincaré Sect。 B 3 (1967), 121-226. MR 35 \#6172.

10. J. Gilbert, On convergence and maximal theorems for some orthogonal series, Trans. Amer。 Math. Soc. 145 (1969), 495-515.

11. S. Helgason, Differential geometry and symmetric spaces, Pure and Appl. Math., vol. 12, Academic Press, New York, 1962. MR 26 \#2986. 
12. L. Hörmander, On the Riesz mèans of spectral functions and eigenfunction expansions for elliptic differential operators, Some Recent Advances in the Basic Sciences, vol. 2 (Proc. Ann. Sci Conf. Belfer Grad. School Sci., Yeshiva Univ., New York, 1965/66), Belfer Graduate School of Science, Yeshiva Univ., New York, 1969, pp. 155-202.

MR 41 \# 2239.

13. - The spectral function of an elliptic operator, Acta Math. 121 (1968), $193-218$.

14. S. Igari and S. Kuratsubo, $A$ sufficient condition for $L^{p}$-multipliers, Pacific J. Math. 38 (1971), 85-88.

15. E. Kogbetliantz, Recherches sur la sommabilité des séries ultrasphériques par la méthode des moyennes arithmétiques, J. Math. Pures Appl. 89 (1924), 107-187.

16. J. Marcinkiewicz, Sur les multiplicateurs des séries de Fourier, Studia Math. 8 (1939), 78-91.

17. B. Muckenhoupt and E. Stein, Classical expansions and their relation to conjugate harmonic functions, Trans. Amer. Math. Soc. 118 (1965), 17-92. MR 33 \#7779.

18. E. Stein, Localization and summability of multiple Fourier series, Acta Math. 100 (1958), 93-147. MR 21 \# 4331.

19. - Topics in harmonic analysis related to the Littlewood-Paley theory, Ann. of Math. Studies, no. 63, Princeton Univ. Press, Princeton, N. J.; Univ. of Tokyo Press, Tokyo, 1970, MR $40 \# 6176$.

20. - Singular integrals and differentiability properties of functions, Princeton Univ. Press, Princeton, N. J., 1971.

21. E. Stein and G. Weiss, Introduction to Fourier analysis on euclidean spaces, Princeton Univ. Press, Princeton, N. J., 1971.

22. G. Szegö, Orthogonal polynomials, Amer. Math. Soc. Colloq. Publ., vol. 23, Amer. Math. Soc., Providence, R. I., 1939. MR 1, 14.

23. N. Ja. Vilenkin, Special functions and the theory of group representations, "Nauka", Moscow, 1965; English transl., Transl. Math. Monographs, vol. 22, Amer. Math. Soc., Providence, R. I., 1968; French transl., Monographies Universitaires de Mathématiques, no. 33, Dunod, Paris, 1969. MR 35 \# 420; MR 37 \# 5429; MR 39 \#4467.

24. N. Weiss, $L^{p}$-estimates for bi-invariant operators on compact Lie groups, Amer. J. Math. (a paraitre).

25. A. Zygmund, Trigonometric series, 2nd ed., Cambridge Univ. Press, New York, 1959. MR $21 \# 6498$.

DEPARTMENT OF MATHEMATICS, UNIVERSITY OF PARIS, CENTRE D'ORSAY, 91 ORSAY, FRANCE 